\title{
RESEARCH
}

Open Access

\section{Decreased nuclear Pten in neural stem cells contributes to deficits in neuronal maturation}

Shin Chung Kang ${ }^{1}$, Ritika Jaini ${ }^{1,2,3}$, Masahiro Hitomi ${ }^{1,2}$, Hyunpil Lee ${ }^{1}$, Nick Sarn ${ }^{1,5}$, Stetson Thacker ${ }^{1,2}$ and Charis Eng $1,2,3,4,5^{*}$

\begin{abstract}
Background: PTEN, a syndromic autism spectrum disorder (ASD) risk gene, is mutated in approximately $10 \%$ of macrocephalic ASD cases. Despite the described genetic association between PTEN and ASD and ensuing studies, we continue to have a limited understanding of how PTEN disruption drives ASD pathogenesis and maintenance.

Methods: We derived neural stem cells (NSCs) from the dentate gyrus (DG) of Pten ${ }^{m 3 m 4}$ mice, a model that recapitulates PTEN-ASD phenotypes. We subsequently characterized the expression of stemness factors, proliferation, and differentiation of neurons and glia in Pten ${ }^{m 3 m 4}$ NSCs using immunofluorescent and immunoblotting approaches. We also measured Creb phosphorylation by Western blot analysis and expression of Creb-regulated genes with qRT-PCR.

Results: The m3m4 mutation decreases Pten localization to the nucleus and its global expression over time. Pten $^{m 3 m 4}$ NSCs exhibit persistent stemness characteristics associated with increased proliferation and a resistance to neuronal maturation during differentiation. Given the increased proliferation of Pten ${ }^{m 3 m 4}$ NSCs, a significant increase in the population of immature neurons relative to mature neurons occurs, an approximately tenfold decrease in the ratio between the homozygous mutant and wildtype. There is an opposite pattern of differentiation in some Pten $^{m 3 m 4}$ glia, specifically an increase in astrocytes. These aberrant differentiation patterns associate with changes in Creb activation in Pten ${ }^{m 3 m 4 / m 3 m 4}$ NSCs. We specifically observed loss of Creb phosphorylation at S133 in Pten $^{\mathrm{m} 3 \mathrm{~m} 4 / \mathrm{m} 3 \mathrm{~m} 4} \mathrm{NSCs}$ and a subsequent decrease in expression of Creb-regulated genes important to neuronal function (i.e., Bdnf). Interestingly, Bdnf treatment is able to partially rescue the stunted neuronal maturation phenotype in Pten ${ }^{m 3 m 4 / m 3 m 4}$ NSCs.

Conclusions: Constitutional disruption of Pten nuclear localization with subsequent global decrease in Pten expression generates abnormal patterns of differentiation, a stunting of neuronal maturation. The propensity of Pten disruption to restrain neurons to a more progenitor-like state may be an important feature contributing to PTEN-ASD pathogenesis.
\end{abstract}

Keywords: PTEN mutation, Neural stem cells, Autism spectrum disorder, Neural development, Neuronal maturation, Creb activation

\footnotetext{
*Correspondence: engc@ccf.org

'Genomic Medicine Institute, Lerner Research Institute, Cleveland Clinic, Cleveland, OH 44195, USA

${ }^{2}$ Cleveland Clinic Lerner College of Medicine, Case Western Reserve University, 9500 Euclid Avenue, Cleveland, OH 44195, USA

Full list of author information is available at the end of the article
}

(c) The Author(s). 2020 Open Access This article is licensed under a Creative Commons Attribution 4.0 International License, which permits use, sharing, adaptation, distribution and reproduction in any medium or format, as long as you give appropriate credit to the original author(s) and the source, provide a link to the Creative Commons licence, and indicate if changes were made. The images or other third party material in this article are included in the article's Creative Commons licence, unless indicated otherwise in a credit line to the material. If material is not included in the article's Creative Commons licence and your intended use is not permitted by statutory regulation or exceeds the permitted use, you will need to obtain permission directly from the copyright holder. To view a copy of this licence, visit http://creativecommons.org/licenses/by/4.0/ The Creative Commons Public Domain Dedication waiver (http://creativecommons.org/publicdomain/zero/1.0/) applies to the data made available in this article, unless otherwise stated in a credit line to the data. 


\section{Background}

Despite the complex etiology of autism spectrum disorder (ASD), it has been established that ASD is highly heritable, where both common and rare genetic variants can contribute to the etiology $[1,2]$. One of the major ASD risk genes encodes the phosphatase and tensin homolog on chromosome ten (PTEN) [3-5]. PTEN germline mutations occur in up to $10 \%$ of macrocephalic ASD, while $\sim 23 \%$ of those with PTEN mutations will receive an ASD diagnosis [3-7]. Individuals with germline mutations in PTEN often have other neurodevelopmental or neurological symptoms as well: developmental delay, mental retardation, learning disability, and epilepsy [6, 7].

The strong genetic association between PTEN and ASD has prompted study of putative molecular mechanisms of disease. These studies have demonstrated the importance of PTEN function and the canonical dysregulation of $\mathrm{PI} 3 \mathrm{~K} / \mathrm{AKT} / \mathrm{mTOR}$ signaling in neuronal development, morphology, and function [8-11]. Furthermore, Pten is known to play a role in neurogenesis within the hippocampus. Knockout of Pten in neural progenitor cells (NPCs) of the hippocampus leads to a high proliferation rate and increased brain volume [1214]. Moreover, PTEN has been shown to have synaptic functions, which are, in part, dependent on neuronal activity [15-17]. Beyond neurons, germline PTEN mutation has also been shown to disrupt astrocyte and oligodendrocyte development and function, indicating a PTEN-dependent complex intercellular crosstalk within the CNS during development [15, 18-22]. Despite these mechanistic insights into PTEN-mediated ASD, little is known about the impact of naturally occurring PTEN mutations, observed in patients and those that impact non-canonical functions (such as the increasingly salient nuclear functions of Pten), on differentiation and development of neuronal stem cells (NSCs). Moreover, ASD likely initiates in utero and/or early during development, making mechanistic insight often difficult to obtain. Therefore, NSCs derived from an established mouse model of PTEN-ASD present a useful approach for investigating ASD pathogenesis during neonatal growth [23, 24].

PTEN downstream canonical signaling typically resides in the cytoplasm. To evaluate how Pten localization and expression regulates neurogenesis and NSC differentiation, we established NSC lines from the dentate gyrus (DG) of Pten ${ }^{m 3 m 4}$ mice. The Pten ${ }^{m 3 m 4}$ mouse is an established model of ASD and exhibits autistic-like traits and behaviors, including sex-dependent increase in social motivation [25]. Pten ${ }^{m 3 m}$ mutants also have deficits in motor coordination yet their motor and spatial learning and spatial and social recognition memory remain intact [25]. Pten ${ }^{m 3 m 4 / m 3 m 4}$ mice present with extreme macrocephaly due to increased brain mass, which, in part, can be explained by the increase in neural soma volumes, activated astrocytes, and aberrant myelin deposits $[20,25,26]$. Follow-up studies on the Pten ${ }^{m 3 m 4}$ phenotypes have demonstrated that oligodendrocyte differentiation, maturation, and myelination are aberrant and dopaminergic signaling is elevated [22, 27]. Using NSCs generated from the Pten ${ }^{m 34}$ brain, we sought to understand the consequence of germline disruption of Pten, resulting in cytoplasmic predominant Pten expression, in influencing NSC differentiation and neuronal development and their concomitant impact on glia.

\section{Methods}

Isolation of neural stem cells (NSCs)

Pten $^{w t / w t}$, Pten ${ }^{w t / m 3 m 4}$, and Pten ${ }^{m 3 m 4 / m 3 m 4}$ mice on a CD1 background were generated and characterized as a model of high-functioning autism as previously described [25]. All experiments were conducted in accordance with protocols approved by Cleveland Clinic's Institutional Animal Care and Use Committee (IACUC). Mice were maintained and euthanized as previously described [24]. We adopted and modified a protocol for the isolation of neural stem cells (NSCs) from dentate gyrus (DG) for adherent cell cultures $[28,29]$. NSCs were isolated from Pten ${ }^{w t / w t}$, Pten $^{m 3 m 4 / m 3 m 4}$, and Pten ${ }^{w t / m 3 m 4}$ mice at postnatal day 20 (P20). Only male mice were used for isolation of wildtype NSCs, whereas a mixture of male and female mice were used for isolation of mutant NSCs. Cerebra were sectioned with a tissue slicer and stored in Hanks' balanced salt solution (HBSS), containing $30 \mathrm{mM}$ glucose, $2 \mathrm{mM}$ HEPES, and $26 \mathrm{mM}$ $\mathrm{NaHCO}_{3}$ and supplemented with $\mathrm{Ca}^{2+}$ and $\mathrm{Mg}^{2+}$. Tissues were digested into a single-cell suspension using the Neural Tissue Dissociation Kit (Miltenyi Biotec $\mathrm{GmbH}$, Germany), following the manufacturer's instructions. NSCs were obtained after a final centrifugation at $200 \mathrm{~g}$ for $5 \mathrm{~min}$ followed by washing with N2 medium (DMEM/F12 with N2 media supplement, and L-glutamine) [28]. For adherent monolayer cell culture, cells were re-suspended in $1 \mathrm{~mL}$ of neurobasal growth media, 1\% (v/v) Gluta-Max, B27 (without vitamin A), $2 \mu \mathrm{g} / \mathrm{ml}$ heparin (MilliporeSigma, Burlington, MA) in the presence of $20 \mathrm{ng} / \mathrm{ml}$ epidermal growth factor (EGF), and $10 \mathrm{ng} / \mathrm{ml}$ fibroblast growth factor 2 (FGF2) beads (StemCultures, NY) and were plated in 96-well plate coated with the $10 \mu \mathrm{g} / \mathrm{ml}$ Poly-L-ornithine (MilliporeSigma) and $5 \mu \mathrm{g} / \mathrm{ml}$ laminin. Growth media was changed every other day. All media and buffers were obtained from ThermoFisher Life Technology (Waltham, MA, USA) unless otherwise specified. 


\section{NSC proliferation and differentiation}

For random differentiation studies, NSCs were cultured in the absence of EGF and FGF2 in neurobasal media for $3,5,7$, and 10 days. Media was replaced every other day with $50 \%$ fresh media. To promote neuronal differentiation, in certain experiments where specified, N2 media supplement was added. Otherwise, NSCs were maintained in EGF $(20 \mathrm{ng} / \mathrm{ml})$ and FGF2 $(10 \mathrm{ng} / \mathrm{ml})$ at $37^{\circ} \mathrm{C}$ and $5 \% \mathrm{CO}_{2}$. Cells were plated at a density of $1 \times$ $10^{5}$ cells $/ \mathrm{ml}$ in poly-L-ornithine/laminin coated 12-well plates in triplicate cultures. Cells were harvested, stained with $0.1 \%$ trypan blue, and counted daily for five days to obtain cell growth rate.

\section{Immunofluorescence}

Immunocytochemistry was performed as described in Lee et al [22]. Briefly, differentiated NSCs were washed with D-PBS and fixed with 100\% ice-cold methanol for 6 min. Fixed NSCs were permeabilized with $0.3 \%$ Triton-X $100 / \mathrm{PBS}$ for $30 \mathrm{~min}$ at room temperature and blocked with $10 \%$ normal goat serum (Vector, Burlingame, CA)/ PBS for $1 \mathrm{~h}$ at room temperature. We utilized primary antibodies as NSC markers: anti-Pax6, anti-Sox2, and anti-c-Myc (Abcam, Cambridge, MA). Anti-Ki67 was used as a proliferation marker. For characterizing differentiation of immature neurons, brain-specific cell subtype markers were used, anti-Tuj1 (Biolegend, San Diego, CA) and anti-doublecortin (DCX) (Cell Signaling Technology). For mature neurons, anti-MAP2 (MilliporeSigma), anti-NeuN (MilliporeSigma), and anti-Syn (Abcam) were used, and for glial cell markers, anti-NG2 (Cell Signaling Technology, Danvers, MA), anti-Olig1 (MilliporeSigma), anti-MBP (MilliporeSigma), and antiGfap (ThermoFisher) were used.

\section{Image analysis and measurements}

Images from ICC were acquired using the Leica TCSSP8-AOBS upright confocal microscope (Leica Microsystems, $\mathrm{GmbH}$, Wetzlar, Germany) at an original magnification of $\times 40$ or $\times 63$, or $\times 100$. The total number of cells in each field was determined by counting DAPIpositive cell nuclei. All images are representative of 3 different areas in each coverslip, taken from at least three biological replicates. Neurite lengths were determined by tracing Tuj1+ neurons in $\times 40$ confocal images using the Simple Neurite Tracer in Image J (FIJI, GitHub). The traces were then analyzed using Sholl Analysis to calculate the number of neurite branches in each image for more than five different areas of each slide. Images of Tuj1+ neurons were traced in more than five neurons on each image taken from three biological replicates. To obtain the ratio of Pten volume in nuclear vs cytoplasmic fractions, ICC for Pten was performed as described above. Confocal microscope images were taken at $\times 100$ magnification, z-stacked, and processed using the Volocity ${ }^{\circ}$ 6.3.0 program (PerkinElmer, Inc, Waltham, MA). In 3D analysis of nuclear and cytoplasmic Pten localization, the number of nuclei containing Pten as well as the total volume of Pten was calculated. All experiments were repeated at least three times in five separate locations on the slides, using 3 biological replicates.

\section{Western blotting}

Differentiated stem cells were harvested, pelleted, and lysed in MPER buffer (ThermoFischer Scientific) with added protease inhibitor cocktails (MilliporeSigma) and incubated for $1 \mathrm{~h}$ on ice. Cell lysates were centrifuged for $15 \mathrm{~min}$ at $16,000 \mathrm{~g}$ at $4{ }^{\circ} \mathrm{C}$. Protein content was quantified using the bicinchoninic acid (BCA) assay 15-50 $\mu \mathrm{g}$ of total protein lysates were separated on a sodium dodecyl sulfate polyacrylamide gel (SDS-PAGE) and analyzed using a standard Western blot protocol. Anti-Pax6, Sox2, and c-Myc antibodies were used as neural stem markers. Nuclear and cytoplasmic Pten fractionation was performed as described [30]. Anti-Hsp90 (Cell Signaling Technology) and anti-Parp (SCT) were used as a cytoplasmic protein and a nuclear protein loading control, respectively. Anti-PTEN (Clone 6H2.1, Cascade Bioscience Inc., Winchester, MA), mTOR (CST), phosphorylated mTOR S2448 D9C2 (CST), total AKT, phosphorylated AKT S473 D9E (CST), and PI3Kinase (ThermoFisher Scientific) were used to examine the PI3K/AKT/mTOR signal pathway. For cell cycle protein assay, anti-CyclinD1, CDK4, and P27 ${ }^{\text {kip1 }}$ were used (Cell Signaling Technology). For the differentiated cells markers, we used anti-Tuj1 (BioLegend, San Diego, CA), NeuN (MilliporeSigma), and c-Fos (Cell Signaling Technology). All antibodies were used at a dilution of 1:1000 for primary and 1:2000 for secondary incubations. For the phosphorylated CREB signaling pathway assay, antiCreb and anti-phosphorylated Creb S133 (MilliporeSigma) were used. Anti-Gapdh (Abcam) and ß-actin (ThermoFisher Scientific) were used as loading controls.

\section{Quantitative real-time PCR (qRT-PCR)}

Total RNA from stem cells and differentiated cells was extracted using NucleoSpin ${ }^{\circ}$ RNA Plus (MachereyNagel, Düren, Germany) according to the manufacturer's instructions. RNA was reverse transcribed using a PrimeScript $^{\text {ma }}$ RT Reagent kit (TAKARA, Kusatsu, Shiga Prefecture, Japan). Quantitative RT-PCR was performed using the $2 \mathrm{x}$ Power $\mathrm{SYBR}^{\circ}$ Green PCR Master Mix (Applied Biosystems, Foster City, CA). Real-time PCR Primers for CREB transcriptional targeted genes such as $B d n f$, Caln1, and, Neurl1b were obtained from IDT (Coralville, IA). Relative gene expression was quantified using the comparative cycle threshold $\left(\mathrm{C}_{\mathrm{T}}\right)$ method 
using Gapdh as the housekeeping gene. The following primers were used: Gapdh (custom synthesis, Thermofisher): F-GGGCTGCCATTTGCAGTGGCAA, RGTGAGTGGAGTCATACTGGAAC, Neurl1b (Assay ID \# Mm.PT.58.43949047), BNDF (Assay ID \# Mm.PT.58.8157970), and Caln1 (Assay ID \# Mm.PT.58.7756375); predesigned qRT-PCR primers were obtained from IDT.

\section{Bdnf rescue of immature neurons}

NSCs grown on Geltrex (growth factor depleted form obtained from Gibco, Waltham, MA) coated coverslips were induced to differentiate by growth factor deprivation in the presence or absence of BDNF (100 $\mathrm{ng} / \mathrm{ml}$ ) for 7 days. Cells were fixed with $3.7 \%(\mathrm{w} / \mathrm{v})$ paraformaldehyde, permeabilized, and blocked with $0.1 \%$ Triton X100, 2\% normal donkey serum in PBS. Fixed cells were immunostained with rabbit anti-Tuj1, mouse anti-NeuN, and rat anti-GFAP antibodies followed by incubation with donkey anti-rabbit, anti-mouse, and anti-rat IgG antibodies labeled with Alexa488, Cy3, and Cy5, respectively. DNA was stained with Hoechst 33342 (100 ng/ml).

After images of each fluorochrome were collected with a fluorescent microscope (Leica DM5000B equipped with a Leica DFC310FX digital camera), each images were processed using an image processing software, Fiji (https://imagej.net/ImageJ). After correcting background signals and shading of illumination of the images accordingly, pixel values of each fluorescence signal were integrated for individual nuclear regions. Signal from dead cells remaining on the plate was eliminated from analysis using graphical mask data region function of KaleidaGraph software (version 4.1, Synergy Software) based on footprint size and intensity of nuclear regions defined by DNA staining. Staining intensities of Tuj1 and NeuN of nuclei were compared between the cultures with or without BDNF by unpaired Wilcoxon-Mann-Whitney rank sum test using KaleidaGraph.

\section{Statistics}

Data are expressed as the mean \pm SD (standard deviation). Student's $t$ tests and one-way ANOVAs were performed, where appropriate, to compare between and among groups, respectively. All Student's $t$ tests were two-tailed and unpaired. Two-way ANOVAs were also employed where appropriate. Tukey-Kramer post hoc testing was used to compare groups and correct for multiple testing following ANOVAs. We also performed Kruskal-Wallis testing, nonparametric ANOVAs, where appropriate with Dunn's multiple comparison post hoc testing. We considered all $p$ values below 0.05 statistically significant. Statistical analyses were performed in GraphPad Prism 8 or KaleidaGraph 4.1.

\section{Results}

Persistence of stemness in NSCs with Pten ${ }^{\text {m3m4/m3m4 }}$ mutations

Pten $^{m 3 m 4}$ mutant neural stem cells (NSCs) cultured from the P20 dentate gyrus showed no gross morphological differences in culture compared to those derived from Pten $^{w t / w t}$ (Additional file 1: Figure S1A). NSCs from all Pten genotypes expressed markers of stemness, namely, Pax6, Sox2, and c-Myc. However, expression of stem cell markers was observed to be elevated in Pten ${ }^{m 3 m 4 / m 3 m 4}$ NSCs, specifically c-Myc $(p$ value $=0.051$; Fig. $1 \mathrm{a}$; Additional file 1: Figure S1B). Overexpression of $\mathrm{c}-\mathrm{Myc}$ is maintained without any decrease until at least 5 days after removal of growth factor (Fig. 1b), in contrast to Pten $^{w t / w t}$ NSCs where c-Myc expression declines beginning at day 3 after growth factor removal ( $p$ value $=$ 0.035; Additional file 1: Figure S1C). Due to this observed persistence of stemness markers in $\mathrm{Pten}^{\mathrm{m} 3 \mathrm{~m} 4 / \mathrm{m} 3 \mathrm{~m} 4}$ NSCs, we evaluated proliferation as a consequence of persistent stemness. Pten ${ }^{m 3 m 4 / m 3 m 4}$ NSCs exhibit higher proliferation rates compared to wildtype NSCs at 5 DIV ( $p$ value $=0.0012 ;$ Fig. 1c). Additionally, Ki67 staining was significantly increased in Pten ${ }^{m 3 m 4 / m 3 m 4}$ relative to wildtype NSCs at 0 DIV (days in vitro, $p$ value $=0.042$ ) and increased in Pten ${ }^{w t / m 3 m 4}$ and $\mathrm{Pten}^{\text {m3m4/m3m4 }}$ relative to wildtype NSCs at $3 \mathrm{DIV}$ ( $p$ value $=0.0039 ; p$ value $<$ 0.0001 , respectively; Fig. 1d; Additional file 1: Figure S1D). However, at $5 \mathrm{DIV}$, persistent Ki67 staining was observed only in Pten ${ }^{m 3 m 4 / m 3 m 4}$ NSCs with no apparent Ki67 expression in Pten ${ }^{w t / m 3 m 4}$ or wildtype NSCs (Fig. 1d). These findings on the prolonged stemness of NSCs derived from $\mathrm{Pten}^{\mathrm{m} 3 \mathrm{~m}^{4}}$ mutants were further supported by observing increased Ccnd1 expression ( $p$ value = $0.014)$ and decreased $\mathrm{p} 27^{\mathrm{kip} 1}$ expression ( $p$ value $=$ 0.039) in Pten ${ }^{m 3 m 4 / m 3 m 4}$ NSCs (Fig. 1e; Additional file 1: Figure S1E).

\section{Nuclear Pten depletion in Pten ${ }^{m 3 m 4 / m 3 m 4}$ NSCs}

$P_{t e n}{ }^{m 3 m}$ mutation is designed to disrupt the nuclear localization-like signals of Pten, leading to predominantly cytoplasmic localization and nuclear depletion of the protein systemically in an adult mouse. In order to check if nuclear depletion of Pten was evident in NSCs, we stained for Pten to evaluate its expression and localization. We observed a decrease in expression and depletion of Pten from the nucleus in mutants (Fig. 2a). Quantification of nuclear and cytoplasmic Pten expression shows an approximately $10 \%$ reduction in homozygous mutant NSCs $(n=5)$ with a 2.5 -fold reduction in the nuclear to cytoplasmic ratio relative to wildtype (Fig. 2b). As random differentiation proceeds, the number of nuclei with Pten expression significantly decreased in Pten $^{m 3 m 4 / m 3 m 4}$ NSCs relative to wildtype NSCs. Quantification of Pten-positive nuclei at 3 days of 

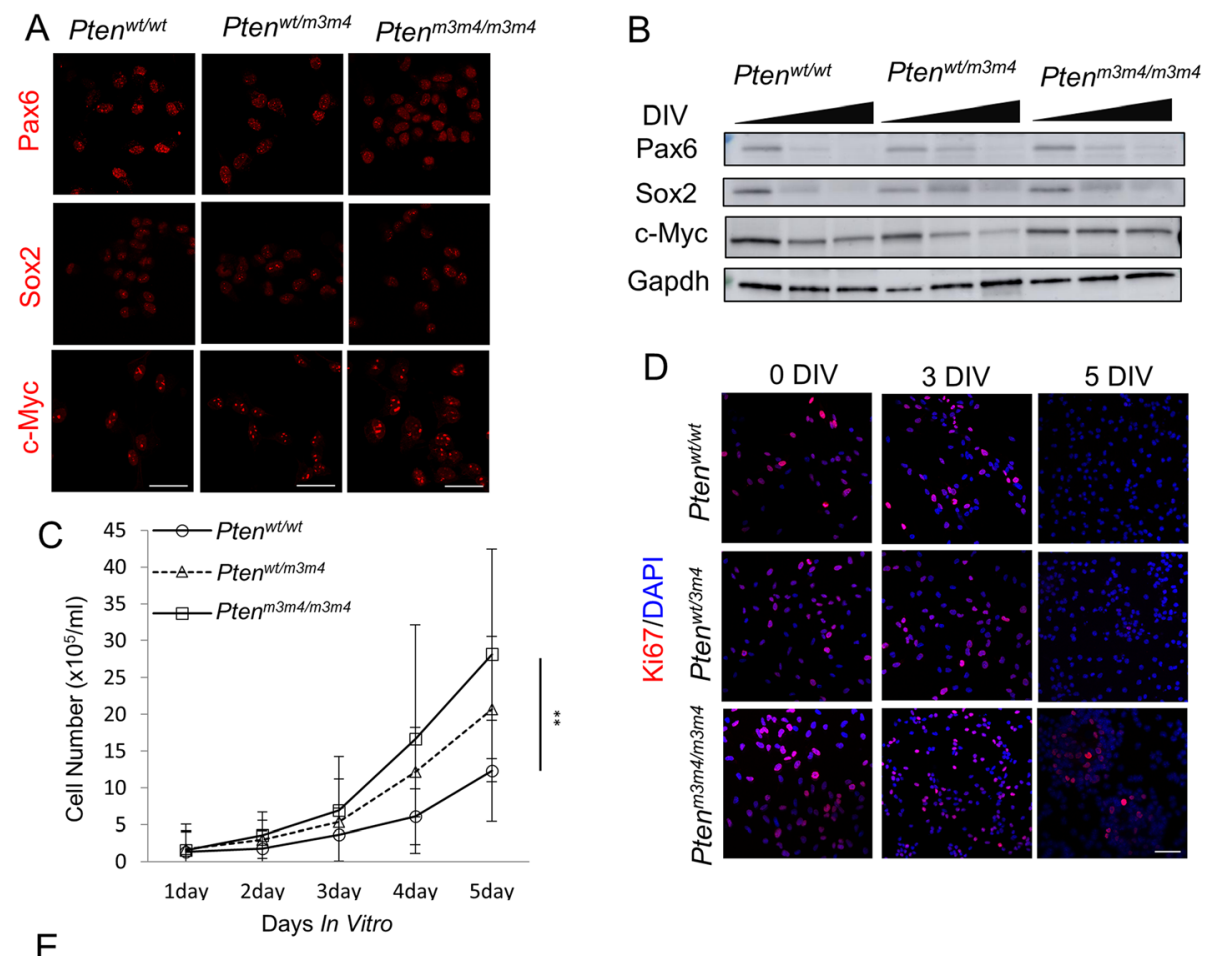

$E$

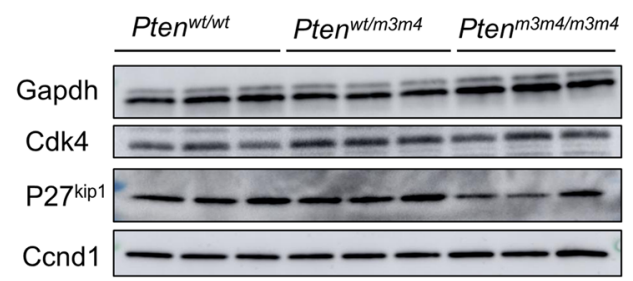

Fig. 1 Characterized neural stem cells (NSCs) derived from Pten ${ }^{m 3 m 4 / m 3 m 4}$ mice have shown slow differentiation. a Immunofluorescence staining for stemness makers Pax6 (top panel), Sox2 (middle panel), and c-Myc (bottom panel) at 1 day in culture with growth factors. b Western blots for stemness markers Pax6, Sox2, and c-Myc over 5 days of random differentiation in the absence of growth factors. Representative blot $(n=3)$. $\mathbf{c}$ Cell counting assay to assess proliferation $(n=5)$. Pten ${ }^{m 3 m 4 / m 3 m 4}$ NSCs show increased proliferation at 5 DIV compared to wildtype as assessed by Kruskal-Wallis testing with Dunn's multiple testing comparison ( $p$ value $=0.0012$ ). $\mathbf{d}$ Immunofluorescence for Ki67 expression at 0, 3, and 5 DIV for all NSC genotypes. e Western blot analysis of undifferentiated NSCS $(n=3)$ for cell cycle progression markers $\left({ }^{*} p\right.$ value $<0.05$; ${ }^{* *} p$ value $<0.01$; **** $p$ value $<0.0001)$

differentiation showed a decreased number of Pten+ nuclei in mutant NSCs compared to Pten ${ }^{w t / w t}$ NSCs (95\% in Pten $^{w t / w t}>85 \%$ in Pten ${ }^{w t / m 3 m 4}>76 \%$ in $\mathrm{Pten}^{\mathrm{m} 3 \mathrm{~m} 4 / \mathrm{m3m4}} ; p$ value $\left.=0.034\right)$. Similarly, quantification of Pten-positive nuclei at 5 days of differentiation showed decreased number of Pten+ nuclei in mutant NSCs compared to Pten ${ }^{w t / w t}$ NSCs (91\% in Pten ${ }^{w t / w t}>$ $50 \%$ in Pten $^{\text {wt } / m 3 m 4}>37 \%$ in Pten $^{\text {m3m4 }}{ }^{2 m 3 m 4} ; p$ value $=$ 0.0051). The difference in Pten-positive nuclei between wildtype and Pten ${ }^{m 3 m 4 / m 3 m 4}$ NSCs increases from 19 to $54 \%$ from day 3 to day 5 of differentiation (Fig. 2c). By visual inspection, the decrease in nuclear Pten expression was further confirmed by Western blot analysis after subcellular fractionation (Fig. 2d). Not only was there a decrease in nuclear Pten expression relative to cytoplasmic expression, but also a global decrease in Pten expression, which we demonstrated by Western blot analysis $(p$ value $=0.0042$; Fig. 2e; Additional file 1: Figure S2A). Moreover, the decline in Pten expression was further exaggerated in Pten mutant NSCs as they differentiate, where Pten expression progressively declined over 5 DIV ( $p$ value $\left._{\text {DIV }}=0.036\right)$ while also showing differences between homozygous mutant and wildtype groups ( $p$ value Pten $=0.021$; Fig. 2 f; Additional file 1: Figure S2B). Given the change in Pten expression and localization, we measured changes in downstream Pi3k/Akt/mTor signaling by Western blot. Pten was again significantly decreased $(p$ value $=0.034)$. As expected, we found significant increases in the phosphorylation of Akt, and mTor in Pten ${ }^{m 3 m 4 / m 3 m 4}$ NSCs, 

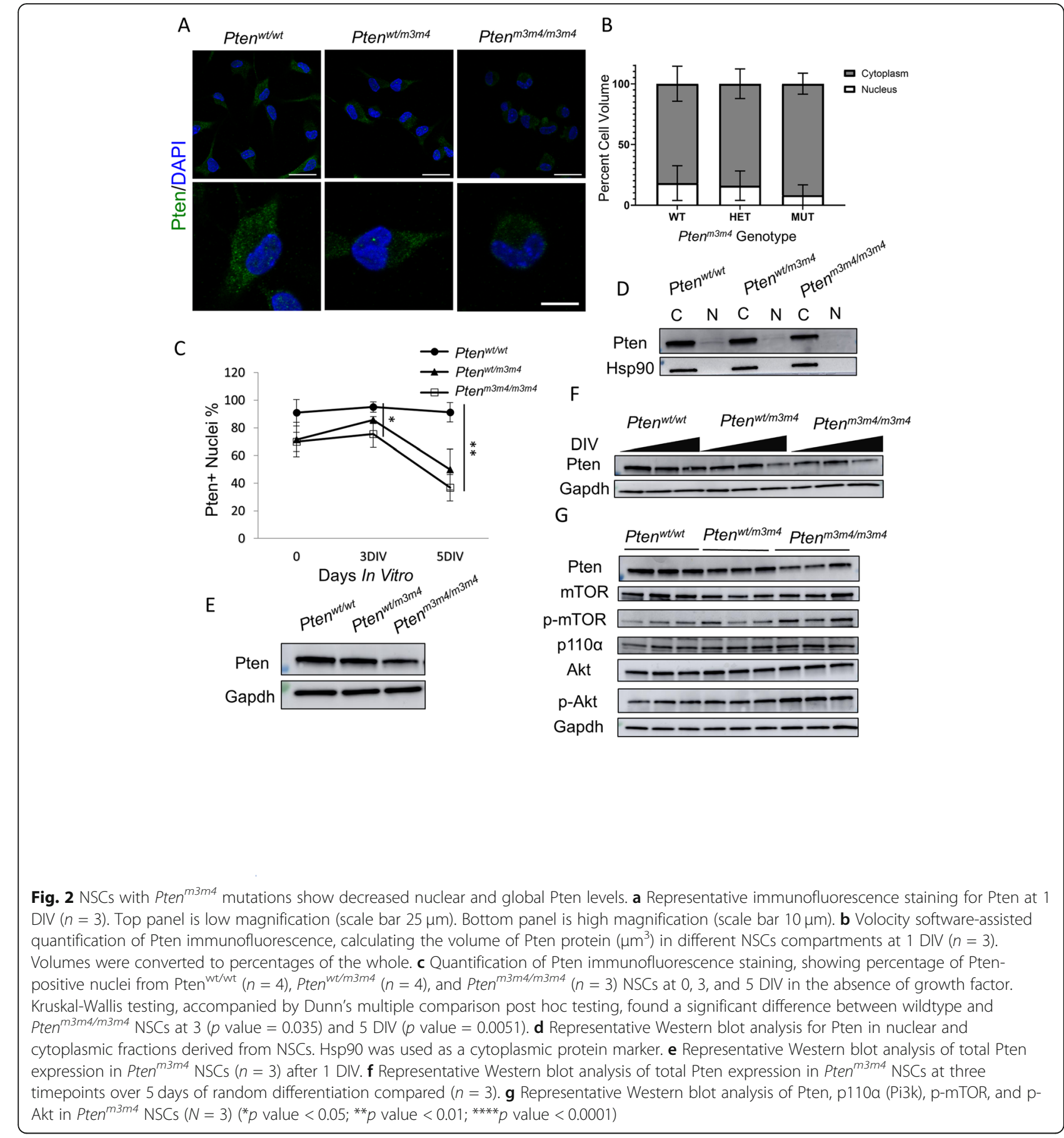

B

reflecting the functional disruption of Pten's lipid phosphatase activity in mutant NSCs ( $p$ values $=0.034$; Fig. 2g). Together, these data illustrate that the Pten ${ }^{\text {m3m4 }}$ mutation results in decreased Pten nuclear localization and decreased Pten overall expression and leads to dysregulation in downstream canonical signaling in NSCs. This decrease in Pten expression and its functional impact on dysregulation of downstream canonical signaling is further exacerbated as these NSCs differentiate.

\section{Deficits in neuronal maturation}

Given the changes in Pten localization and expression in concert with the persistent stemness of mutant NSCs, we decided to monitor the neuronal differentiation of $P_{t e n}{ }^{m 3 m 4}$ NSCs. Thus, we allowed for 5 days of random differentiation and then stained for markers of immature (Tuj1 and Dcx) and mature (Map2 and NeuN) neurons. We found a significant increase in the numbers of Tuj1positive $(p$ value $=0.0051)$ and Dcx-positive $(p$ value $=$ 
0.033) immature neurons in Pten ${ }^{\mathrm{m} 3 \mathrm{~m} 4 / \mathrm{m} 3 \mathrm{~m} 4}$ NSC cultures. In contrast, expression of the mature neuronal marker NeuN was significantly decreased in differentiated $P$ ten ${ }^{m 3 m 4 / m 3 m 4}$ NSCs $(p$ value $=0.015)$. No change was observed in expression of Map2 between any of the genotypes. Interestingly, a significantly higher number of Tuj1-positive neurons were present among differentiated Pten ${ }^{m 3 m 4 / m 3 m 4}$ NSCs compared to the wildtype NSC cultures (Fig. 3a, b). These findings indicate that Pten $^{m 3 m 4 / m 3 m 4}$ NSCs differentiate into immature neurons earlier and more aggressively than their wildtype counterparts but subsequently delay maturation into NeuN-expressing neurons, which may be a function of the prolonged stemness of mutant NSCs.

To underscore that the observation of reduced NeuN expression in the mutant NSCs was indicative of stunted maturation, we stained for the pan-presynaptic marker synaptophysin at 7 and 10 DIV across all genotypes (Additional file 1: Figure S3B). We found that Syn expression significantly increased between 7 and 10 DIV in wildtype NSCs $(p$ value $=0.034)$ but not in heterozygous or homozygous mutant NSCs. However, we did not find
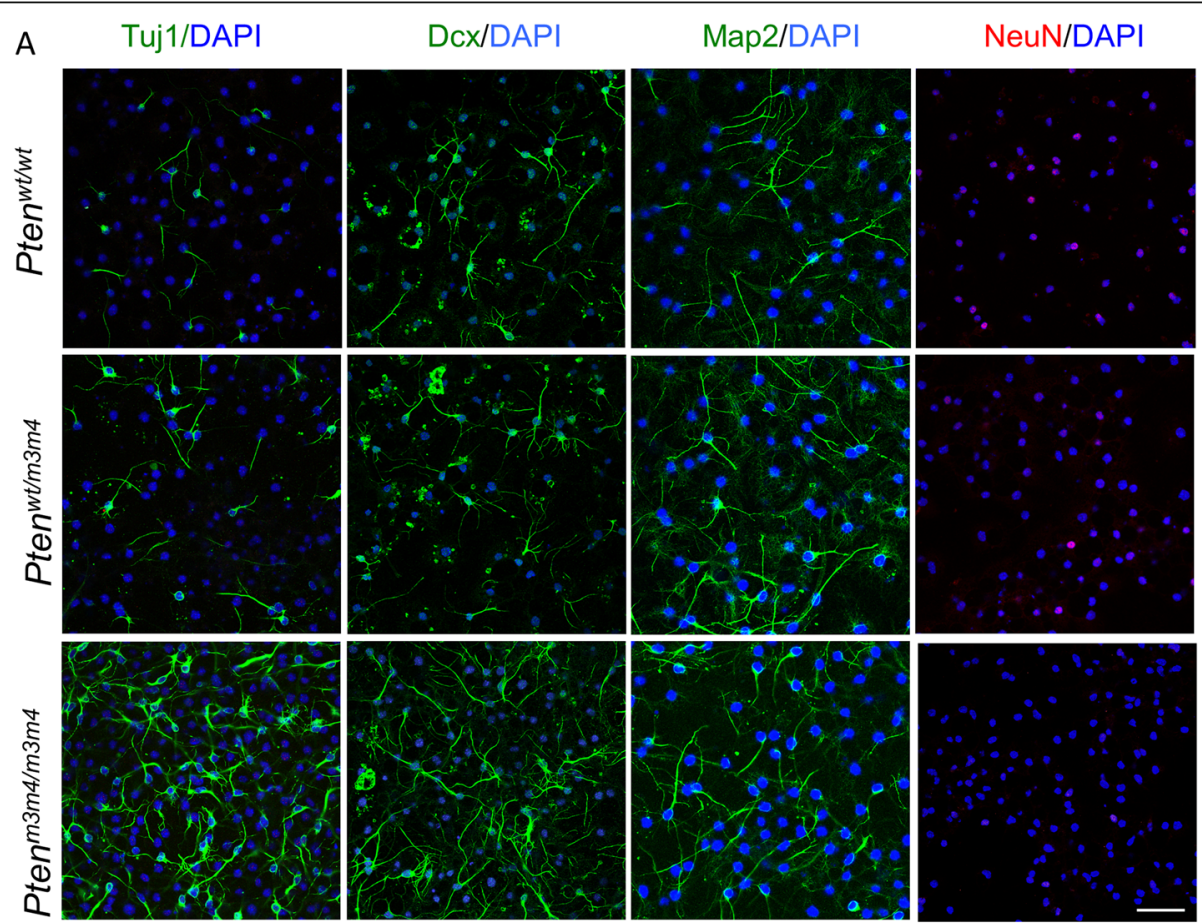

B

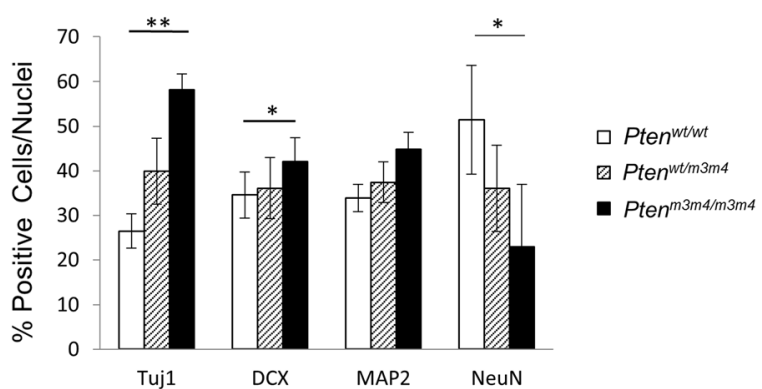

Fig. 3 Pten ${ }^{m 3 m 4}$ NSCs differentiation halts after becoming immature neurons. a Representative immunofluorescence staining for neuronal markers Tuj1 (green, $n=5$ ), Dcx (green, $n=3$ ), Map2 (green, $n=3$ ), and NeuN (red, $n=3$ ), showing increased presence of immature neurons in Pten $^{m 3 m 4 / m 3 m 4}$ NSCs compared to wildtype NSCs when cultured in the absence of specific lineage-driving growth factors for 10 days. NeuN (mature neuron marker) expression is significantly lower in Pten ${ }^{m 3 m 4 / m 3 m 4}$ NSCs compared to their wildtype counterparts. b Quantification of immunofluorescence staining of cells expressing neuronal markers (Tuj1, Dcx, Map2, and NeuN) normalized to total number of nuclei (DAPI) per frame. Kruskal-Wallis testing, accompanied by Dunn's multiple comparison testing, finding a significant increase in Tuj $1(p$ value $=0.0051)$ and $\operatorname{Dcx}(p$ value $=0.033)$ and a significant decrease in NeuN $(p$ value $=0.015)$ comparing Pten ${ }^{m 3 m 4 / m 3 m 4}$ and wildtype NSCs $\left({ }^{*} p\right.$ value $<0.05 ; *^{* *} p$ value $<0.01)$ 
significant differences in Syn expression normalized to cell number among different genotypes (Additional file 1: Figure S3B). Although the pattern of Syn expression does not clearly show deficits in synapse formation in the mutants, it does show a lack of developmental increase in synapse formation from 7 to 10 DIV in culture.

To understand the nature of deficits in neuronal differentiation in Pten ${ }^{m 3 m 4 / m 3 m 4}$ NSCs, we decided to monitor the respective immature and mature population of neurons over a longer course of differentiation (Fig. 4a-d). Consistent with our previous findings (Fig. 3), we found an increase in the number of Tuj1-positive cells in Pten ${ }^{m 3 m 4 / m 3 m 4}$ NSCs at earlier differentiation timepoints, specifically 5 and 7 DIV ( $p$ values $=0.050$ and 0.013 , respectively). However, even with extended differentiation periods, Pten $^{m 3 m 4 / m 3 m 4}$ NSCs maintained an increased number of Tuj1-positive neurons compared to both Pten $^{m 3 m 4 / w t}$ and Pten ${ }^{w t / w t}$ NSCs (Fig. 4a, b). This was
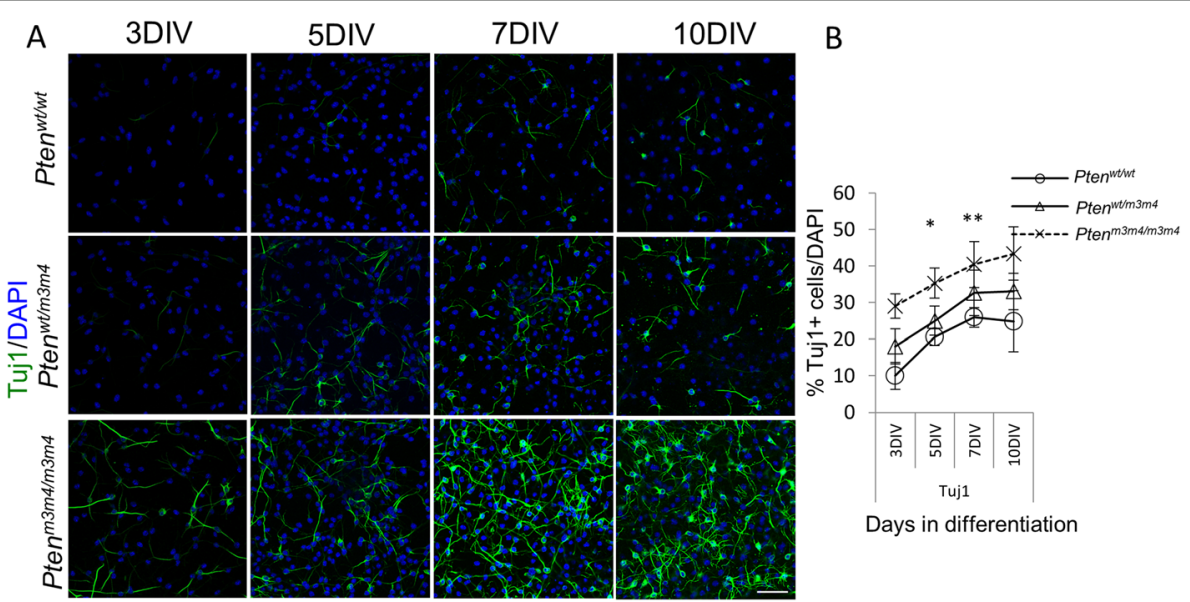

Days in differentiation

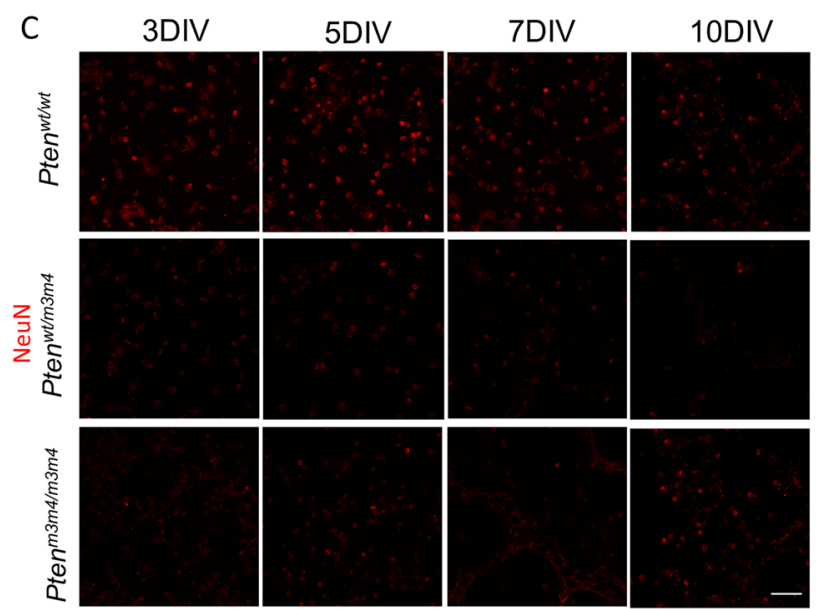

D

$\mathrm{E}$

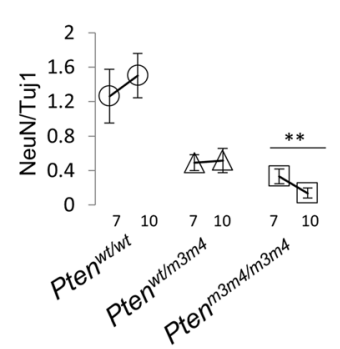

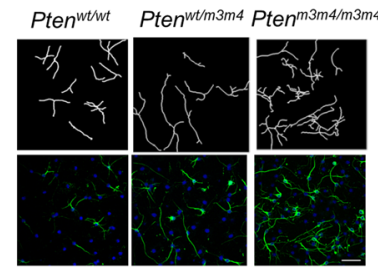

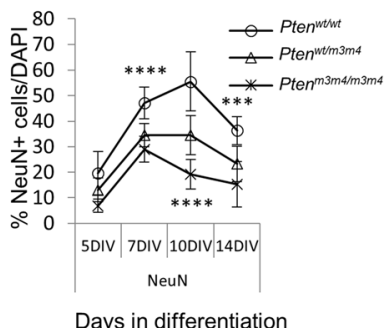

G

$\mathrm{H}$
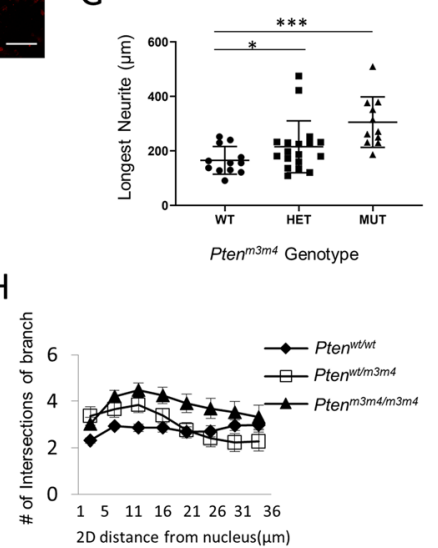

Fig. 4 (See legend on next page.) 
(See figure on previous page.)

Fig. 4 Pten $^{m 3 m 4}$ NSC differentiation is precocious yet abortive, leading to increased immature neuron population. a Representative

immunofluorescence staining defines immature neuron population (Tuj1+ cell in green: DAPI+ nuclei in blue) at 3, 5, 7, and 10 days in vitro in the absence of growth factors. $\mathbf{b}$ Quantification of staining in $\mathbf{a}$, where a significant increase in Tuj1+ neurons at 5 DIV $(p$ value $=0.05)$ and 7 DIV ( $p$ value $=0.0013$ ) comparing Pten ${ }^{\mathrm{m} 3 \mathrm{~m} 4 / \mathrm{m} 3 \mathrm{~m} 4}$ to Pten ${ }^{\text {wt } / w t}$ as assessed by Kruskal-Wallis testing with Dunn's multiple comparison testing. $\mathbf{c}$ Immunofluorescence staining for mature neuron (NeuN+ cells in red: DAPI+ nuclei in blue) population in culture for 5, 7, 10, and 14 DIV in the absence of growth factor. d Quantification of mature neurons in c (NeuN+ cells). Kruskal-Wallis testing with Dunn's multiple comparison testing found significant decreases in NeuN+ cells at 7 ( $p$ value $=<0.0001), 10(p$ value $=<0.0001)$, and 14 DIV $(p$ value $=0.003)$ between $P$ ten ${ }^{\mathrm{m} 3 \mathrm{~m} 4 / \mathrm{m} 3 \mathrm{~m} 4}$ and wildtype NSCs and significant decreases in NeuN+ cells at 7 ( $p$ value $=0.018)$ and 14 DIV $(p$ value $=0.029)$ between Pten ${ }^{\text {wt } / \mathrm{m} 3 \mathrm{~m} 4}$ and wildtype NSCs. e NeuN+ cells to Tuj1+ cells at 7 and 10 DIV $(n=3)$. Mann Whitney $U$ testing found a significant different between the 7 and 10 DIV NeuN+/Tuj1+ ratios in Pten ${ }^{\mathrm{m} 3 \mathrm{~m} / \mathrm{m} 3 \mathrm{~m} 4}$ NSCs ( $p$ value $\left.=0.0012\right)$. $\mathbf{f}$ Tuj1+ neurites showing arbored branched intersections of neurites $(n=3)$. g Simple neurite tracing quantification by Image J (Sholl analysis), showing increased mean length of longest NSC neurite in Tuj1+ cells derived from Pten ${ }^{m 3 m 4 / m 3 m 4}$ NSCs (means wt vs het vs mut: $165.4 \mu \mathrm{m}$ vs $215.5 \mu \mathrm{m}$ vs $305.7 \mu \mathrm{m}$ ), indicating a significant increase between Pten ${ }^{\mathrm{m} 3 \mathrm{~m} 4 / \mathrm{m} 3 \mathrm{~m} 4}$ and wildtype NSCs ( $p$ value $=0.0006$ ) and between Pten ${ }^{w t / m 3 m 4}$ and wildtype NSCs ( $p$ value $\left.=0.020\right)$. $\mathbf{h}$ Quantification of branch intersection of neurites, showing consistently increased branch complexity in Pten ${ }^{m 3 m 4 / m 3 m 4}$ NSCs at increasing distance from nucleus ${ }^{*} p$ value $<0.05$; ${ }^{*} p$ value $<0.01 ;{ }^{* * *} p$ value $\left.<0.0001\right)$

correlated with significantly decreased numbers of NeuN-positive cells in Pten ${ }^{m 3 m 4}$ mutant NSC cultures over 14 days of differentiation, where NeuN-positive cells were decreased in Pten ${ }^{m 3 m 4 / m 3 m 4}$ versus wildtype at 7,10 , and 14 DIV ( $p$ values $=<0.0001,<0.0001$, and 0.0030, respectively; Fig. 4c, d). We observed that while Pten wildtype NSCs progressively differentiated into mature neurons over a time course of 14 days in culture, NSCs with $\mathrm{Pten}^{\mathrm{m} 3 m 4}$ mutations showed some NeuN-positive staining at day 7 in culture, but eventually failed to develop a robust population of NeuNpositive neurons. Cultures were terminated at 14 days of differentiation because we observed increased cell death at this timepoint. Consistent with these observations, we demonstrate that the ratio of NeuNpositive to Tuj1-positive cells increases in wildtype NSCs but decreases in homozygous mutant NSCs ( $p$ value $=0.0012$; Fig. 4e). Moreover, the $\operatorname{Pten}^{m 3 m 4 / m 3 m 4}$ NSCs exhibit an increase in both length and complexity of neurites compared to wildtype, a morphological phenotype indicative of immature status (Fig. $4 \mathrm{f}-\mathrm{h}$ ). There are significant increases in the average length of the longest neurite in both Pten ${ }^{w t / m 3 m 4}$ ( $p$ value $=$ $0.020)$ and Pten ${ }^{m 3 m 4 / m 3 m 4}$ ( $p$ value $\left.=0.0006\right)$ NSCs at 10 DIV relative to wildtype NSCs (Fig. 4g). These findings collectively suggest that neuronal differentiation of Pten ${ }^{m 3 m 4 / m 3 m 4}$ NSCs is precocious yet stunted, resulting in a larger pool of immature neurons. Pten ${ }^{m 3 m 4 / m 3 m 4}$ NSCs rapidly differentiate into immature neurons in greater number than wildtype NSCs, but the increased pool of immature neurons fail to reach maturity at the same rate as wildtype NSCs.

\section{Limited response in $P_{t e n}{ }^{m 3 m 4 / m 3 m 4}$ NSCs to neuronal differentiation induction}

To investigate functional implications of a defect in maturation of Pten ${ }^{m 3 m 4 / m 3 m 4}$ NSCs into mature neurons, we investigated their neuronal activity. We measured c-Fos expression, a proxy for neuronal activity, by Western blot in differentiated Pten ${ }^{m 3 m 4 / m 3 m 4}$ NSCs. We found that c-Fos expression was decreased in differentiated Pten ${ }^{m 3 m 4 / m 3 m 4}$ NSCs relative to both differentiated wildtype and $P_{t e n}{ }^{w t / m 3 m 4}$ NSCs, suggesting that neuronal activity in culture correlates with the population of mature neurons $(p$ value $=0.0022)$. This observation is supported by the trends in NeuN and Tuj1 expression, which show a significant decrease $(p$ value $=0.0042)$ and increase $(p$ value $=0.003)$ in differentiated Pten $^{m 3 m 4 / m 3 m 4}$ NSCs, respectively (Fig. 5a). Next, we sought to assess whether neuronal growth factor-driven NSC differentiation would alter the efficiency of $\mathrm{Pten}^{\text {m3m4/m3m4 }}$ NSC differentiation into mature neurons. We found that addition of N2 supplement to NSCs cultures did, in fact, increase NeuN expression in mutant NSCs (assessed by visual inspection), suggesting an increased drive towards maturation in the population of NSCs (Fig. 5b, third panel); however, the Pten ${ }^{m 3 m 4 / m 3 m 4}$ expression of NeuN was still significantly decreased compared to wildtype after 10 days of differentiation driven by growth factors $(p$ value $=0.0066)$. The expression of c-Fos responded to N2 treatment, increasing in all genotype groups, though showing the lowest expression levels in the homozygous mutant NSCs (Fig. 5b). This was underscored by the finding of no-significant difference in NeuN expression across genotypes and timepoints of differentiation ( $p$ value $=0.095$ ). Moreover, $\mathrm{N} 2$ treatment provoked a significant increase in Tuj1 expression in mutant NSCs ( $p$ value $=0.0007$ ). These results underscore the finding that Pten ${ }^{m 3 m 4 / m 3 m 4}$ NSCs prematurely arrest maturation because decreased neuronal activity is indicative of reduced mature neurons in cultures.

\section{Patterns of glial differentiation}

Given the precocious yet abortive pattern of neuronal differentiation, and our previous work describing the white matter abnormalities and astrogliosis in the 

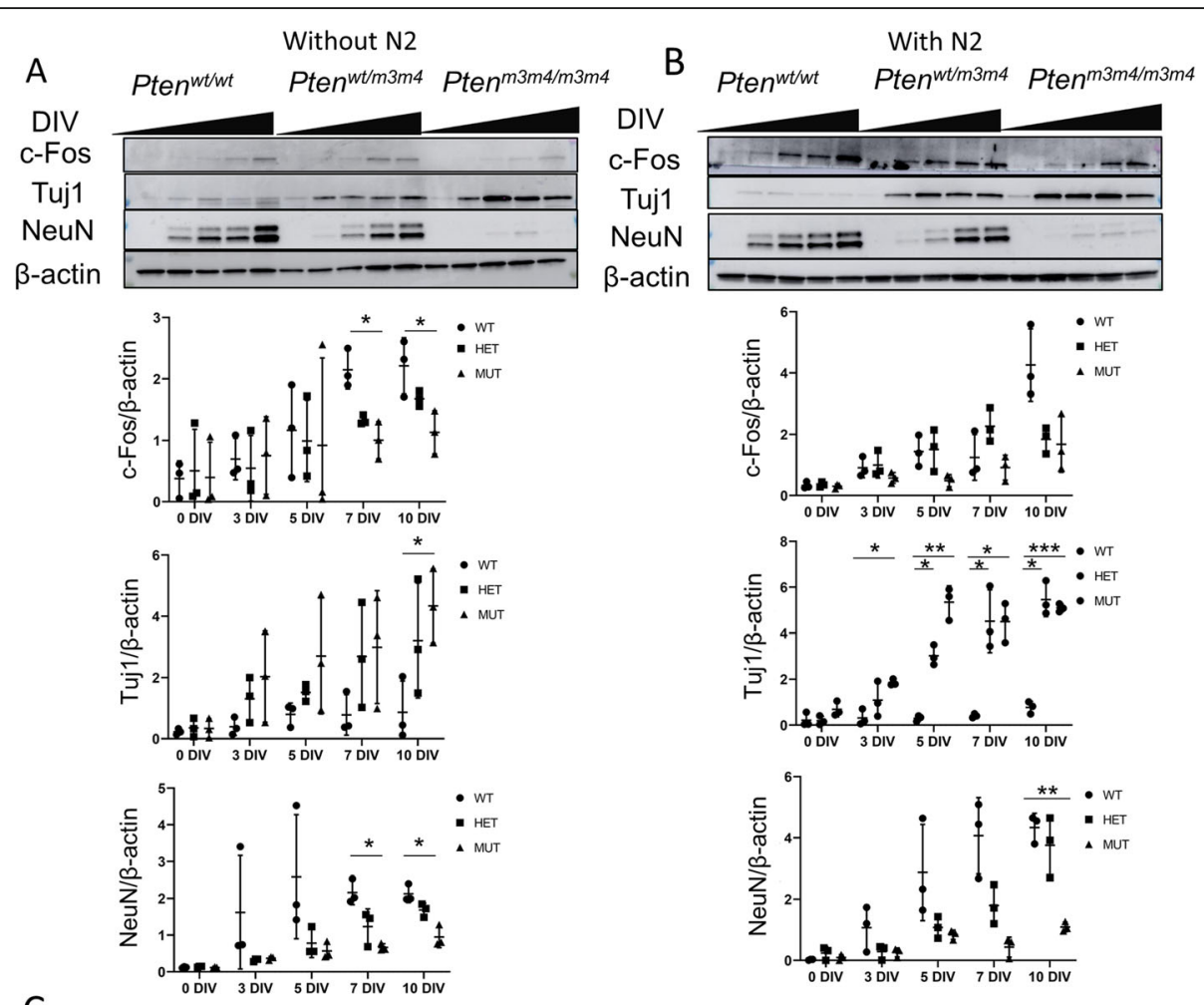

C
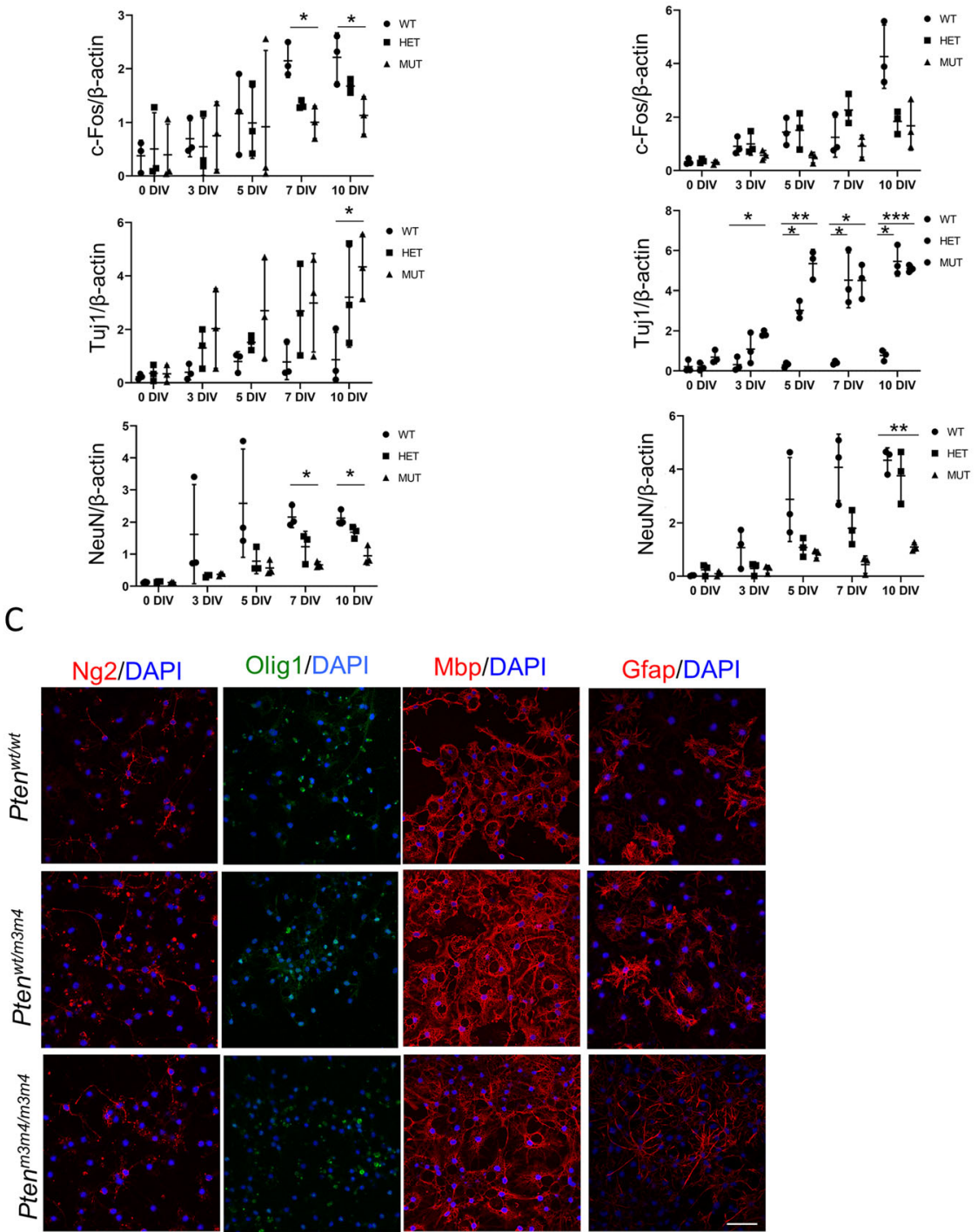

Fig. 5 Pten ${ }^{m 3 m 4}$ NSCs exhibit changes in neuronal activity and glia differentiation. a Western blot analysis $(n=3)$ assessing c-Fos, Tuj1, and NeuN expression in differentiated NSCS without specific lineage-driving growth factors (top panel) with quantification (bottom panel). Two-way ANOVA with Tukey-Kramer post hoc testing found significant decrease in c-Fos and NeuN in Pten ${ }^{\mathrm{m} 3 \mathrm{~m} 4 / \mathrm{m} 3 \mathrm{~m} 4}$ versus wildtype at 7 DIV $(p$ value $=0.025$; 0.021 ) and 10 DIV ( $p$ value $=0.034 ; 0.014)$. A significant increase in Tuj1 between Pten ${ }^{\mathrm{m} 3 \mathrm{~m} 4 / \mathrm{m} 3 \mathrm{~m} 4}$ and wildtype at 10 DIV was also observed $(p$ value $=0.043$. Two-way ANOVA with Tukey-Kramer post hoc testing found significant decrease in NeuN in Pten ${ }^{\mathrm{m} 3 \mathrm{~m} 4 / \mathrm{m} 3 \mathrm{~m} 4}$ versus wildtype at 10

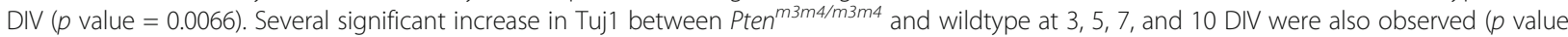
$=0.017 ; 0.0099 ; 0.025 ; 0.0002$, respectively). $\mathbf{b}$ Western blot analysis $(n=3)$ shows c-Fos, Tuj1, and NeuN expression in differentiated NSCs treated with N2 supplement (top panel) with quantification (bottom panel). Densitometric quantification for specific protein expression is normalized to ß-actin. c Immunofluorescent staining at 10 DIV without growth factors for oligodendrocyte precursors cell (OPC), oligodendrocyte (OL), and myelination markers: Ng2 (red), Olig1 (green), Mbp (red), and Gfap (red), respectively ( ${ }^{*} p$ value $<0.05 ;{ }^{* *} p$ value $<0.01 ;{ }^{* * *} p$ value $<0.001$; ${ }^{* * * *} p$ value $<0.0001$ ) 
Pten $^{m 3 m 4}$ mouse [20, 22, 25], we were interested in any associated changes in oligodendrocyte (OL) lineage and astrocyte differentiation in $\mathrm{Pten}^{m 3 m 4}$ NSC cultures. After 10 days of random differentiation, we stained for markers of OL and astrocyte differentiation: Ng2, Olig1, $\mathrm{Mbp}$, and Gfap. We found no changes in Ng2, Olig1, and $\mathrm{Mbp}$ expression suggesting no differences in the number of oligodendrocyte precursor cells (OPCs) or OLs, respectively (Fig. 5c, d). Although we did not find any expression differences in OL makers, we found an increase in Gfap expression as well as morphological changes in astrocytes differentiated from Pten $^{m 3 m 4 / m 3 m 4}$ NSC, indicating astrogliosis ( $p$ value $=0.032$; Fig. $5 \mathrm{c}$; Additional file 1: Figure S4A). These data highlight the effect that Pten disruption has on glial differentiation in addition to the effects on neuronal differentiation. The increase in astrocyte proliferation and change in morphology is striking.

\section{Activation of Creb correlates with differentiation}

Previous work has demonstrated a correlation between Pten loss and Creb activation in NSCs [10]. Thus, we sought to examine whether the relationship between the m3m4 mutation in Pten and Creb activation in NSCs. As such, we differentiated Pten ${ }^{m 3 m 4}$ NSCs with or without N2 supplement and then measured Creb phosphorylation at S133, a residue subject to dephosphorylation by Pten [10]. Surprisingly, we observed a decline in (almost absence of) Creb phosphorylation in both Pten $^{w t / m 3 m 4}$ and Pten ${ }^{m 3 m 4 / m 3 m 4}$ NSCs independent of N2 supplement treatment (Fig. 6a, b). The N2 supplement treatment did slightly alter the pattern of Creb phosphorylation in wildtype NSCs, causing it to peak at day three, followed by a progressive decline, as opposed to peaking at day five followed by a steep decline in expression where differentiation was induced without N2 supplement (Fig. 6a, b). As part of a validation effort to confirm changes in Creb activation, we measured relative expression of transcripts known to be regulated by Creb (Bdnf, Caln1, Neur1b, and Rest) at three timepoints $(0,7$, and 10 DIV). Consistent with the findings of the Western blot analysis, we observed significant decreases in the gene expression of Creb-regulated, neurodevelopmentally relevant targets. Moreover, we found the dysregulation occurred more broadly at the undifferentiated timepoint for NSCs, where three of four targets were significantly changed versus only 2 at differentiated timepoints. Interestingly, the dysregulation of Creb targets was not comprehensive. For instance, despite showing lower expression on average in the mutant NSC genotypes, Rest was not significantly decreased in expression compared to wildtype (Fig. 6c). Collectively, these results illustrate that changes in Creb activation are at least temporally associated with the observed deficits in differentiation in Pten mutant NSCs.

\section{Bdnf partially restores neuronal maturation in mutant NSCs}

The finding of decreased Bdnf expression subsequent to the decrease in Creb activation may be related to the NeuN phenotype due to the well-described role of Bdnf in promoting neurogenesis. To assess whether Bdnf treatment can rescue the decrease in NeuN expression in the homozygous mutant NSCs, we treated $\operatorname{Pten}^{m 3 m 4}$ NSCs with Bdnf and stained for Tuj1, NeuN, and Gfap (Fig. 7a). We found that that Bdnf treatment significantly increased NeuN expression in the heterozygous and homozygous mutant NSCs ( $p$ value $<0.0001$ ); however, there rescue was only partial in effect not returning mutant NeuN to wildtype-like levels (Fig. 7b). These data illustrate that decreased Bdnf expression in Pten ${ }^{m 3 m 4}$ NSCs is responsible, in part, for their stunted neuronal maturation.

\section{Discussion}

Consistent with previous studies, our data show an increase in cellular proliferation and enhanced neurogenesis in NSCs with depleted Pten expression [14, 31-35]. In contrast, we show that a constitutive decrease in Pten expression, prominently in the nucleus, as observed in the Pten ${ }^{m 3 m 4}$ model, is associated with stunted neuronal maturation, despite enhanced neurogenesis, after Pten $^{m 3 m 4}$ NSCs become immature yet post-mitotic neurons. The resulting accumulation of immature neurons is rapid and striking, where few mutant neurons progress to complete maturity, marked by NeuN expression (Figs. 3 and 4). The precocious yet abortive pattern of neuronal differentiation may be explained by the prolonged elevation in the expression of stem markers and subsequently the division/self-renewal capacity of Pten $^{m 3 m 4}$ mutant NSCs. Some of this differentiation pattern may also be due to the surprising lack of Creb activation in mutant NSCs, which also decrease Bdnf levels (Figs. 6 and 7). Although stunted maturation is evident only in the neuronal lineage, there appears to be some differential effects on glia: apparently unaffected OL differentiation and a clear increase in astrocyte proliferation and activation (Figs. 5 and 7). These findings are largely consistent with reported phenotypic observations in adult Pten $^{m 3 m 4}$ mice, where behavioral features reminiscent of high-functioning autism are observed, where morphological and physiological changes in neurons and astrocytes are observed without changes in cell numbers [25].

In our previous work, we characterized OPC differentiation isolated from the developing cortex of newborn $\mathrm{Pten}^{\mathrm{m} 3 \mathrm{~m} 4}$ mice. Interestingly, we found precocious, 

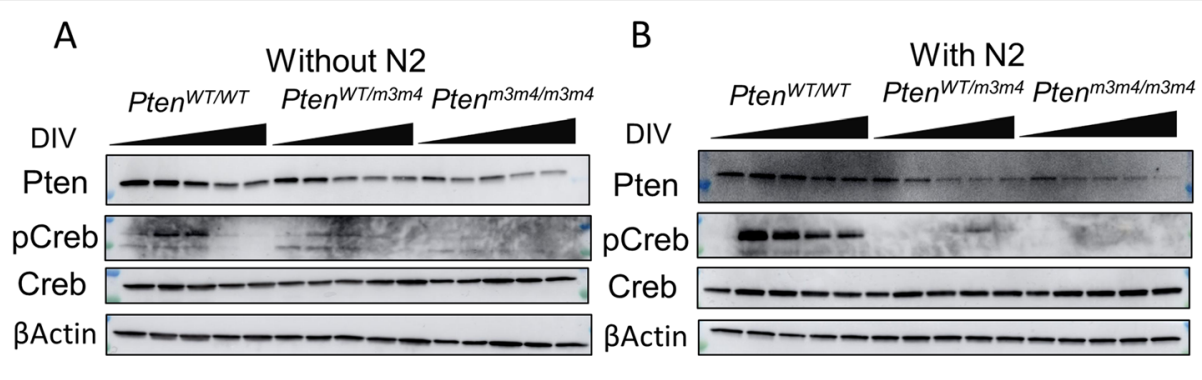

C

Bdnf

Caln1

Neur1b

Rest
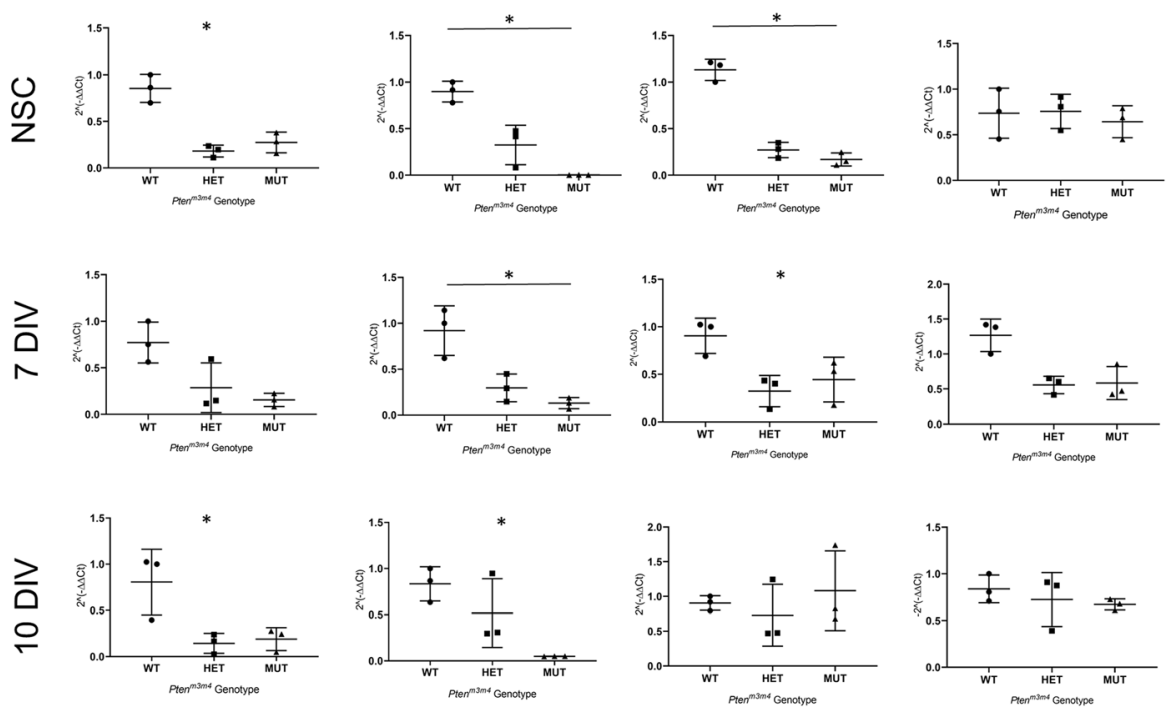

Fig. 6 Changes in Creb activation in Pten ${ }^{m 3 m 4}$ NSCs. a Western blot analysis of differentiated NSCs at 0, 3, 5, 7, and 10 DIV (depicted as rising black bar) without N2 growth factors. b Western blot analysis of differentiated NSCs at 0, 3, 5, 7, and 10 DIV (depicted as rising black bar) with N2 supplement $(n=3)$. c Transcript expression of Creb-regulated genes (Bdnf, Caln1, Nerl1b, and Rest) quantified by qRT-PCR at three timepoints: NSC, 7 DIV, and 10 DIV. For the NSC timepoint, Kruskal-Wallis testing found a significant difference in expression in Bdnf ( $p$ value $=0.025)$, Caln $1(p$ value $=0.0036$ ), and Neurib ( $p$ value $=0.011$ ) among genotype groups, where Dunn's multiple comparison testing identified a significant difference between mutant and wildtype expression of Caln1 $(p$ value $=0.022)$ and Neur1b $(p$ value $=0.034)$. For the 7 DIV timepoint, KruskalWallis test found a significant difference in expression in Caln $1(p$ value $=0.011)$ and Neur1b $(p$ value $=0.025)$ among genotype groups, where Dunn's multiple comparison testing identified a significant difference between mutant and wildtype expression of Caln1 ( $p$ value $=0.034)$. For the 10 DIV timepoint, Kruskal-Wallis test found a significant difference in expression in Bdnf ( $p$ value $=0.025)$ and Caln $1(p$ value $=0.025)$ among genotype groups. Three biological replicates $(n=3)$ were performed for each genotype group at each timepoint and relative transcript expression was measured in $2^{-(\Delta \Delta C t)}\left({ }^{*} p\right.$ value $\left.<0.05\right)$

enhanced proliferation and increased myelin production; however, the myelin deposition observed is abnormal, generating bleb-like structures in the OLs, which often occur adjacent to, rather than, circumferentially, engulfing axons. In fact, we found that myelin thickness significantly decreased around mutant axons, which showed increased caliber [22]. It is possible that our study failed to observe a white matter phenotype because of the differing cellular contexts of the two studies. The OPC differentiation and dysmyelination in the Pten $^{m 3 m 4}$ model $[22,25]$ was observed in OPCs isolated from P2 cortices versus the NSCs from this study isolated from P20 DG. However, it remains unclear why the $\mathrm{m} 3 \mathrm{~m} 4$ mutation did not generate a white matter phenotype in NSCs in vitro. One can speculate cell-cell interactions are critically important in a 3-dimensional context in enacting the white matter phenotype.

In contrast to OL differentiation, astrocyte differentiation observed in mutant NSCs faithfully recapitulates the astrogliosis phenotype observed in the Pten $^{m 3 m 4 / m 3 m 4}$ brain [25]. Importantly, the NSC differentiation data show that the $\mathrm{m} 3 \mathrm{~m} 4$ mutation can affect both neuronal and glial lineages and in a fashion that is likely specific to each lineage.

The deficits in neuronal maturation in $\mathrm{Pten}^{m 3 m 4}$ NSCs may, in part, be explained by surprising changes in Creb activation in $\mathrm{Pten}^{m 3 m 4}$ NSCs, and its subsequent effect on Bdnf expression. A previous study demonstrated that 


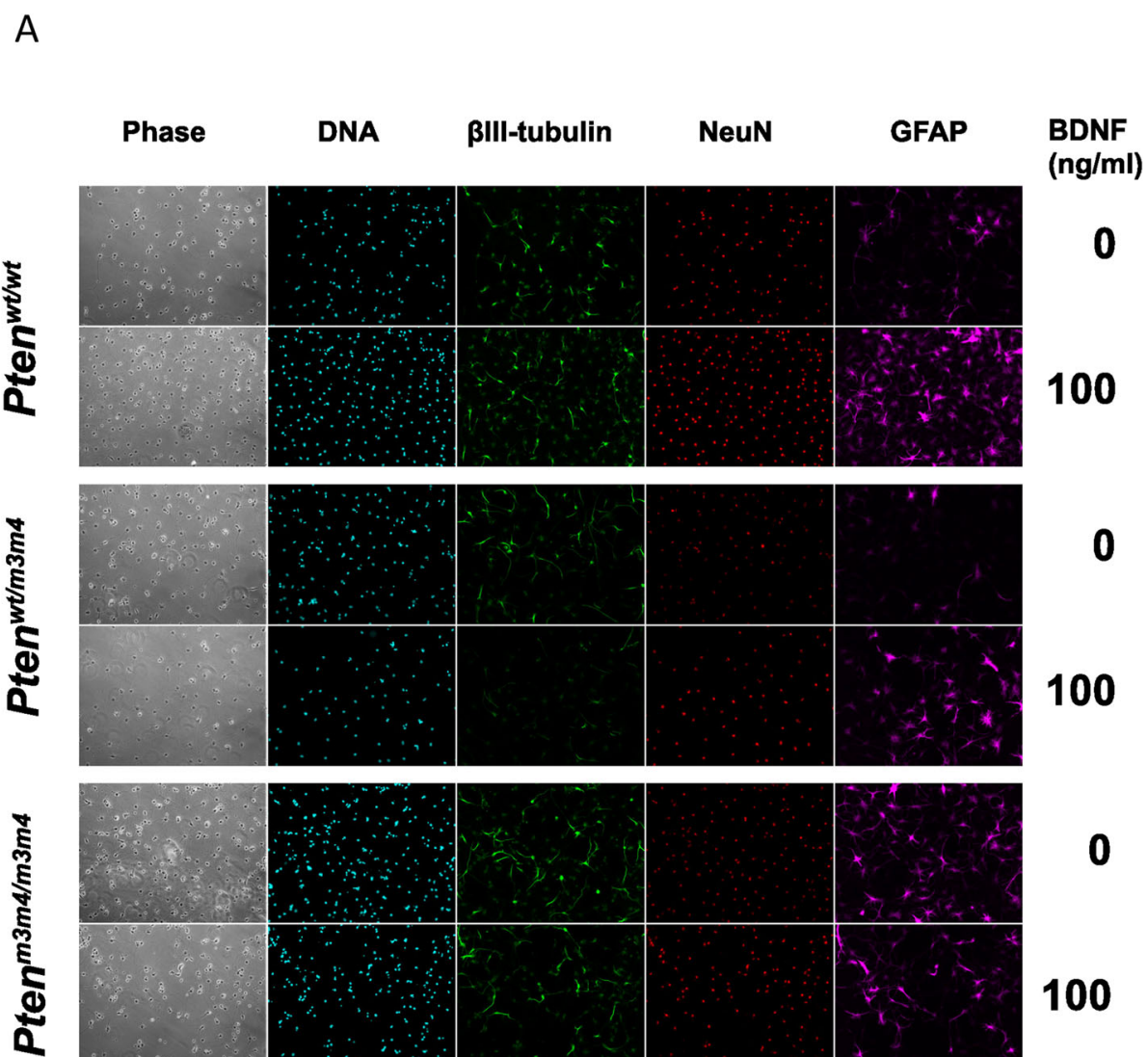

B

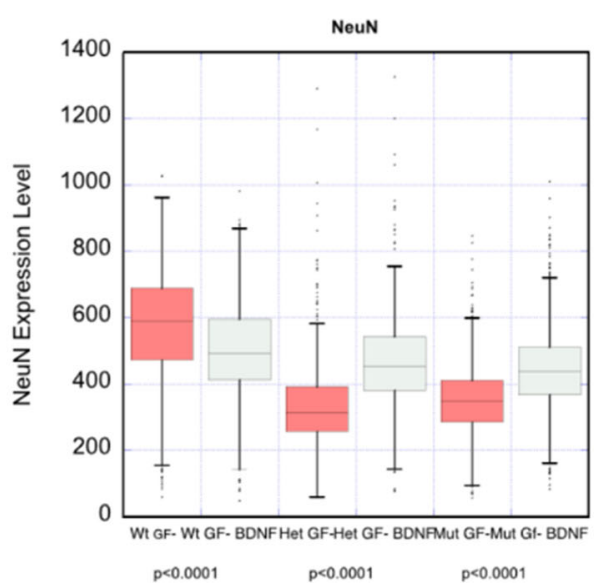

Fig. 7 BDNF rescue of neuronal maturation in Pten ${ }^{m 3 m 4}$ NSCs. a Immunofluorescent staining for Tuj1, NeuN, Gfap, and nuclear DNA with or without $100 \mathrm{ng} / \mu \mathrm{L}$ BDNF treatment. b Cell-based quantification of NeuN staining across all Pten ${ }^{\mathrm{m} 3 \mathrm{~m} 4}$ NSC genotypes

the protein phosphatase activity of PTEN on S133 of CREB is critical in the regulation of neuronal differentiation, where Pten knockdown or disruption of the protein phosphatase activity of Pten increases the population of Tuj1-positive neurons during differentiation [10]. Our findings are consistent in that Pten disruption results in an increased pool of Tuj1-positive neurons; however, our data on the phosphorylation of
Creb is divergent. We see a paradoxical reduction of Creb phosphorylation relative to Pten expression in the nucleus (Fig. 6a, b). It is possible that the decline in the nuclear localization of Pten would induce another phosphatase to decrease Creb phosphorylation or that the $\mathrm{m} 3 \mathrm{~m} 4$ Pten mutant may regulate cell signaling in a fashion that indirectly prevents increased Creb phosphorylation or that rampantly increased Pi3k/Akt signaling 
induces a feedback mechanism that reverses Creb activation. What is clear is that the lack of Creb activation associated with a decrease in Bdnf expression and that Bdnf treatment can partially rescue neuronal maturation in Pten ${ }^{m m 4}$ NSCs. This highlights the need for further research into the effects of Creb phosphorylation on neuronal maturation and the non-canonical roles of Pten in regulating this process.

An important distinction between our data and Lyu and colleagues' data [10] is that we quantitatively examined differentiation of mature neurons (i.e., the NeuNpositive and Syn-positive populations), whereas their primary quantitative measure of neuronal differentiation was Tuj1-positive staining, a marker of post-mitotic yet immature neurons. This precludes a direct comparison with our findings. The mature neuron population in their model may be different, and this potential difference may explain the difference in the Creb activation data. Overall, our data suggest that the role of Pten in neuronal differentiation can be critically yet subtly tuned by different mutations, especially when comparing a cell-specific, conditional knockout model to a constitutive model of missense mutation. This implicates many of the non-canonical functions of Pten, which generally occur in the nucleus. Additionally, our data are consistent with the Lyu and colleagues' study in that we find an increase in neurite length in differentiated mutant NSCs, but diverge in that we find changes in glia, specifically astrocytes differentiation where they did not.

In this study, we have identified a more complex, specified role for Pten in neurogenesis and neuronal maturation. We illustrate that Pten is more than a negative regulator of neurogenesis and neuronal maturation, and its participation in these processes is likely modulated by mutation type. A wealth of research has established that Pten is an important regulator of normal neuronal development, checking proliferation, promoting differentiation, and balancing maturation [36-38]. Our study expands upon these findings, while illustrating the nuanced effects that non-canonical Pten functions, especially in the nucleus, likely have on neuronal differentiation.

\section{Limitations}

Although NSCs are an excellent model to study ASD pathogenesis, and Pten makes for a straightforward genetic model of ASD, our study is limited in scope in that only one Pten genotype was investigated, albeit, this genotype represents the common endpoint of a major subset of non-canonical signaling. Moreover, this endpoint is largely representative of a group of different patient mutations (i.e., those that disrupt Pten nuclear localization and stability and occur at residues distant from the catalytic motif). Despite the Pten ${ }^{m 3 m 4}$ model's faithful recapitulation of the clinical phenotypes of macrocephalic ASD as a component of PHTS, it is difficult to parse which phenotypes are attributable to the changes in localization versus the changes in expression of Pten versus changes in function specific to the $\mathrm{m} 3 \mathrm{~m} 4$ mutation itself. Ultimately, these questions can only be answered by comprehensively modeling patient mutations in iPSCs and animal models.

\section{Conclusion}

Ultimately, we show that $\mathrm{m} 3 \mathrm{~m} 4$ mutation of Pten decreases nuclear localization and global expression of Pten in NSCs, a pattern that is exacerbated by differentiation (Fig. 2). Next, we demonstrate persistent stemness or a proclivity for progenitor status in $\mathrm{Pten}^{m 3 m 4 / m 3 m 4}$ NSCs, which is marked by maintained upregulation of cMyc (Fig. 1a, b). Subsequently, Pten ${ }^{m 3 m 4 / m 3 m 4}$ NSCs exhibit increased proliferation (Fig 1c-e). Consistent with the stemness and proliferation results, we observe an accumulation of immature neurons from differentiated Pten $^{m 3 m 4 / m 3 m 4}$ NSCs that fail to reach maturity (Figs. 3 and 4). The deficits in neuronal differentiation are accompanied by deficits in glial differentiation, specifically astrogliosis (Fig. 5c). To simply illustrate the pattern of neurogenesis and gliogenesis markers over differentiation time, we have constructed a summary schematic (Additional file 1: Figure S5). Changes in differentiation may be partially mediated by changes in Creb activation in Pten ${ }^{m 3 m 4 / m 3 m 4}$ NSCs (Fig. 6). This is underscored by the ability of Creb-regulated Bdnf to partially rescue neuronal maturation in mutant NSCs. Because we have already shown that the Pten ${ }^{m 3 m 4 / m 3 m 4}$ neural transcriptome affects genes responsible for idiopathic ASD [39], this current study expands our knowledge concerning PTEN-ASD pathogenesis, and possibly all ASD, defining a critical role for non-canonical, likely nuclear, Pten function in neurogenesis, gliogenesis, and neuronal maturation.

\section{Supplementary information}

Supplementary information accompanies this paper at https://doi.org/10. 1186/s13229-020-00337-2.

Additional file 1: Supplementary Information. Decreased nuclear Pten in neural stem cells contributes to deficits in neuronal maturation. Figure S1. Neural stem cells (NSCs) derived from Pten ${ }^{m 3 m 4}$ mice have higher stemness characteristics. a NSCs derived from dentate gyrus (DG) of wildtype (left, $n=3$ ), Pten ${ }^{w t / m 3 m 4}$ (center, $n=4$ ), and Pten ${ }^{m 3 m 4 / m 3 m 4}$ (right, $n=4)$ mice had similar morphology after 1 day and 5 days $(n=3)$ of in vitro growth in cell cultures containing growth factors (EGF and FGF2). Images shown in 20X. Pten ${ }^{m 3 m 4}$ mutant NSCs showed a lack of contact inhibition (white arrow). b Quantification of c-Myc staining in Figure 1a, showing a significant increase in c-Myc expression in the homozygous mutant relative to wildtype ( $p$-value $=0.051)$. c Quantification of c-Myc expression at 5 DIV finding a significant increase in expression for Pten $^{m 3 m 4 / m 3 m 4}$ NSCs compared to wildtype ( $p$-value $\left.=0.035\right)$ and Pten $^{\text {wt } / m 3 m 4}$ ( $p$-value $\left.=0.0042\right)$ NSCs $(p$-value $=0.035$ ) as assessed by one- 
way ANOVA with Tukey-Kramer post hoc testing. d Quantification of Ki67 immunofluorescence at 0 and 3 DIV, finding a significant difference in Ki67+ nuclei between Pten ${ }^{m 3 m 4 / m 3 m 4}$ and wildtype NSC at 0 ( $p$-value $=$ $0.036)$ and 3 ( $p$-value $<0.0001)$ DIV as assessed by one-way ANOVA with Tukey-Kramer post hoc testing. There was also a significant increase in Ki67+ nuclei between Pten ${ }^{\omega t / m 3 m 4}$ and wildtype NSCs at 3 DIV ( $p$-value $=$ 0.0039 ) as assessed by one-way ANOVA with Tukey-Kramer post hoc testing e Western blot analysis of undifferentiated NSCs $(n=3)$, showing increased Ccnd $1(p$-value $=0.014)$ and P27kip1 $(p$-value $=0.039)$ expression in Pten ${ }^{\mathrm{m} 3 \mathrm{~m} / \mathrm{m} / \mathrm{m} 3 \mathrm{~m} 4}$ NSCs compared to wildtype NSCs as assessed by ANOVA with Tukey-Kramer post hoc testing. A significant difference in expression of Ccnd 1 between $\operatorname{Pten}^{\mathrm{m} 3 \mathrm{~m}} 4 / \mathrm{m} 3 \mathrm{~m} 4 \mathrm{~A}$ and Pten $^{\text {wt/m3m4 }}$ NSCs ( $p$-value $=0.011$ ) was also found as assessed by ANOVA with Tukey-Kramer post hoc testing $\left({ }^{*} p\right.$-value $<0.05$; ${ }^{*} p$-value $<0.01$; ***** $p$-value $<0.0001)$. Figure S2. NSCs with Pten ${ }^{m 3 m 4}$ mutations show decreased nuclear and global Pten levels. a Densitometry quantification of ratio of Pten expression normalized to Gapdh in Pten ${ }^{m 3 m 4 / m 3 m 4}$ NSCs vs Pten ${ }^{\text {wt } / \text { wt }}$ NSCs at 1 DIV ( $p$-value $=0.0042$ ). b Densitometric quantification of Western blot analyses of Pten expression normalized to Gapdh over 5 days of random differentiation. Two-way ANOVA testing found differences in Pten expression ( $p$-value $=0.021$ ) and between the time points ( $p$-value $=0.036$ ), where Pten expression explains $38 \%$ of the variance and time in culture explains $15 \%$. No interaction was found between factors (Pten expression and DIV) was found. c Densitometric quantification of Western blot analysis of Pten, p110a (Pi3k), p-mTor, and p-Akt expression in Pten ${ }^{m 34}$ NSCs. Kruskal-Wallis testing, accompanied by Dunn's multiple comparison testing, found a significant decrease in Pten $(p$-value $=0.034)$ and a significant increase in p-mTOR ( $p$-value $=$ $0.034)$ and $p$-Akt ( $p$-value $=0.034)$ in Pten ${ }^{m 3 m 4 / m 3 m 4}$ versus wildtype NSCs. Figure S3. Synapthophysin staining on Pten ${ }^{m 3 m 4}$ NSCs. a High magnification representative image of Syn+ (green) cells at seven DIV with DAPI (blue). Scale bar $=10 \mu \mathrm{m}$. b Top panel: Low magnification representative image of Syn+ (green) cells at 10 DIV with DAPI (blue). Bottom panel: High magnification of circled image in top panel. Scale bar $=20 \mu \mathrm{m}$. c Quantification percent Syn+ cells normalized to DAPI expression. Only significant difference between wildtype Syn expression at seven and 10 DIV ( $p$-value $<0.05$ ). Scale bar $=20 \mu \mathrm{m}$. Figure S4. Quantitative view of gliagenesis in Pten ${ }^{m 3 m 4}$ NSCs. a Quantification of gliagenesis staining data in Figure 5, including Ng2, Olig1, Mbp, and Gfap. Gfap shows a significant difference in expression between wildtype and homozygous mutant by integrated density or stain area normalized to cell number ( $p$-value $=0.032$ and 0.017 , respectively). $\mathbf{b}$ Gfap staining trace analysis followed by stain area and perimeter calculations.

\section{Abbreviations}

ASD: Autism spectrum disorder; PTEN: Phosphatase and tensin homolog on chromosome ten; DG: Dentate gyrus; NSC: Neural stem cells; OPC: Oligodendrocyte progenitor cell; OL: Oligodendrocyte

\section{Acknowledgements}

We are grateful to Blake Chaffee and Weelic Chong for helping to establish an NSC isolation protocol for the Eng lab. CE is the Sondra J. and Stephen R. Hardis Endowed Chair of Cancer Genomic Medicine at the Cleveland Clinic and is an ACS Clinical Research Professor.

\section{Authors' contributions}

Conception and design: RJ, ST, HL, NS, CE. Experimentation and data acquisition: SCK, MH. Interpretation of data: SCK, RJ, ST, MH, HL, NS, CE. Drafting of manuscript: SCK, ST, RJ, CE. Critical revisions of manuscript: ST, RJ, $\mathrm{MH}, \mathrm{CE}$. Final approval of manuscript: All authors

\section{Funding}

This study was funded, in part, by the Ambrose Monell Foundation and the Zacconi Program of PTEN Research Excellence.

\section{Availability of data and materials}

The datasets for this study are available from the corresponding author on reasonable request.

\section{Ethics approval and consent to participate}

This study was approved by Cleveland Clinic Lerner Research Institute's IACUC.

\section{Consent for publication}

Not applicable.

\section{Competing interests}

The authors declare that they have no competing interests.

\section{Author details}

${ }^{1}$ Genomic Medicine Institute, Lerner Research Institute, Cleveland Clinic, Cleveland, OH 44195, USA. ${ }^{2}$ Cleveland Clinic Lerner College of Medicine, Case Western Reserve University, 9500 Euclid Avenue, Cleveland, OH 44195, USA. ${ }^{3}$ Case Comprehensive Cancer Center, Case Western Reserve University School of Medicine, Cleveland, OH 44106, USA. ${ }^{4}$ Taussig Cancer Institute, Cleveland Clinic, Cleveland, OH 44195, USA. ${ }^{5}$ Department of Genetics and Genome Sciences, Case Western Reserve University School of Medicine, Cleveland, OH 44106, USA.

\section{Received: 30 September 2019 Accepted: 13 April 2020}

Published online: 01 June 2020

\section{References}

1. De Rubeis S, Buxbaum JD. Genetics and genomics of autism spectrum disorder: embracing complexity. Hum Mol Genet. 2015 Oct 15;24(R1): R24-31.

2. Sullivan PF, Geschwind DH. Defining the genetic, genomic, cellular, and diagnostic architectures of psychiatric disorders. Cell. 2019 Mar 21;177(1):162-83.

3. Butler MG, Dasouki MJ, Zhou X-P, Talebizadeh Z, Brown M, Takahashi TN, et al. Subset of individuals with autism spectrum disorders and extreme macrocephaly associated with germline PTEN tumour suppressor gene mutations. J Med Genet. 2005 Apr;42(4):318-21.

4. McBride KL, Varga EA, Pastore MT, Prior TW, Manickam K, Atkin JF, et al. Confirmation study of PTEN mutations among individuals with autism or developmental delays/mental retardation and macrocephaly. Autism Res Off J Int Soc Autism Res. 2010 Jun;3(3):137-41.

5. O'Roak BJ, Vives L, Fu W, Egertson JD, Stanaway IB, Phelps IG, et al. Multiplex targeted sequencing identifies recurrently mutated genes in autism spectrum disorders. Science. 2012 Dec 21:338(6114):1619-22.

6. Tan M-H, Mester JL, Ngeow J, Rybicki LA, Orloff MS, Eng C. Lifetime cancer risks in individuals with germline PTEN mutations. Clin Cancer Res Off J Am Assoc Cancer Res. 2012 Jan 15;18(2):400-7.

7. Yehia L, Eng C. Largescale population genomics versus deep phenotyping: Brute force or elegant pragmatism towards precision medicine. NPJ Genomic Med. 2019;4:6.

8. Stiles BL. Phosphatase and tensin homologue deleted on chromosome 10: extending its PTENtacles. Int J Biochem Cell Biol. 2009 Apr;41(4):757-61.

9. Zhou J, Blundell J, Ogawa S, Kwon C-H, Zhang W, Sinton C, et al. Pharmacological inhibition of mTORC1 suppresses anatomical, cellular, and behavioral abnormalities in neural-specific Pten knock-out mice. J Neurosci Off J Soc Neurosci. 2009 Feb 11;29(6):1773-83.

10. Lyu J, Yu X, He L, Cheng T, Zhou J, Cheng C, et al. The protein phosphatase activity of PTEN is essential for regulating neural stem cell differentiation. Mol Brain. 2015 Apr 18;8:26.

11. Tilot AK, Frazier TW, Eng C. Balancing proliferation and connectivity in PTENassociated autism spectrum disorder. Neurother J Am Soc Exp Neurother. 2015 Jul;12(3):609-19.

12. Kwon C-H, Luikart BW, Powell CM, Zhou J, Matheny SA, Zhang W, et al. Pten regulates neuronal arborization and social interaction in mice. Neuron. 2006 May 4;50(3):377-88.

13. Ogawa S, Kwon C-H, Zhou J, Koovakkattu D, Parada LF, Sinton CM. A seizure-prone phenotype is associated with altered free-running rhythm in Pten mutant mice. Brain Res. 2007 Sep 7;1168:112-23.

14. Amiri A, Cho W, Zhou J, Birnbaum SG, Sinton CM, McKay RM, et al. Pten deletion in adult hippocampal neural stem/progenitor cells causes cellular abnormalities and alters neurogenesis. J Neurosci Off J Soc Neurosci. 2012 Apr 25;32(17):5880-90.

15. Fraser MM, Bayazitov IT, Zakharenko SS, Baker SJ. Phosphatase and tensin homolog, deleted on chromosome 10 deficiency in brain causes defects in synaptic structure, transmission and plasticity, and myelination abnormalities. Neuroscience. 2008 Jan 24;151(2):476-88. 
16. Jurado S, Benoist M, Lario A, Knafo S, Petrok CN, Esteban JA. PTEN is recruited to the postsynaptic terminal for NMDA receptor-dependent longterm depression. EMBO J. 2010 Aug 18;29(16):2827-40.

17. Takeuchi K, Gertner MJ, Zhou J, Parada LF, Bennett MVL, Zukin RS Dysregulation of synaptic plasticity precedes appearance of morphological defects in a Pten conditional knockout mouse model of autism. Proc Natl Acad Sci U S A. 2013 Mar 19;110(12):4738-43.

18. Harrington EP, Zhao C, Fancy SPJ, Kaing S, Franklin RJM, Rowitch DH. Oligodendrocyte PTEN is required for myelin and axonal integrity, not remyelination. Ann Neurol. 2010 Nov;68(5):703-16.

19. Maire CL, Ramkissoon S, Hayashi M, Haidar S, Ramkissoon L, DiTomaso E, et al. Pten loss in Olig2 expressing neural progenitor cells and oligodendrocytes leads to interneuron dysplasia and leukodystrophy. Stem Cells Dayt Ohio. 2014 Jan;32(1):313-26.

20. Frazier TW, Embacher R, Tilot AK, Koenig K, Mester J, Eng C. Molecular and phenotypic abnormalities in individuals with germline heterozygous PTEN mutations and autism. Mol Psychiatry. 2015 Sep;20(9):1132-8.

21. González-Fernández E, Jeong H-K, Fukaya M, Kim H, Khawaja RR, Srivastava IN, et al. PTEN negatively regulates the cell lineage progression from NG2+ glial progenitor to oligodendrocyte via mTOR-independent signaling. eLife. 2018;20:7.

22. Lee H, Thacker S, Sarn N, Dutta R, Eng C. Constitutional mislocalization of Pten drives precocious maturation in oligodendrocytes and aberrant myelination in model of autism spectrum disorder. Transl Psychiatry. 2019 17;9(1):13.

23. Lancaster MA, Renner M, Martin C-A, Wenzel D, Bicknell LS, Hurles ME, et al. Cerebral organoids model human brain development and microcephaly. Nature. 2013 Sep 19;501(7467):373-9.

24. Kelava I, Lancaster MA. Stem cell models of human brain development. Cell Stem Cell. 2016 02;18(6):736-748.

25. Tilot AK, Gaugler MK, Yu Q, Romigh T, Yu W, Miller RH, et al. Germline disruption of Pten localization causes enhanced sex-dependent social motivation and increased glial production. Hum Mol Genet. 2014 Jun 15;23(12):3212-27.

26. Mester JL, Tilot AK, Rybicki LA, Frazier TW, Eng C. Analysis of prevalence and degree of macrocephaly in patients with germline PTEN mutations and of brain weight in Pten knock-in murine model. Eur J Hum Genet EJHG. 2011 Jul;19(7):763-8.

27. He X, Thacker S, Romigh T, Yu Q, Frazier TW, Eng C. Cytoplasm-predominant Pten associates with increased region-specific brain tyrosine hydroxylase and dopamine D2 receptors in mouse model with autistic traits. Mol Autism. 2015;6:63.

28. Guo W, Patzlaff NE, Jobe EM, Zhao X. Isolation of multipotent neural stem or progenitor cells from both the dentate gyrus and subventricular zone of a single adult mouse. Nat Protoc. 2012 Nov;7(11):2005-12.

29. Walker TL, Kempermann G. One mouse, two cultures: isolation and culture of adult neural stem cells from the two neurogenic zones of individual mice. J Vis Exp JoVE. 2014 Feb 25;84:e51225.

30. Gagnon KT, Li L, Janowski BA, Corey DR. Analysis of nuclear RNA interference in human cells by subcellular fractionation and Argonaute loading. Nat Protoc. 2014 Sep;9(9):2045-60.

31. Groszer M, Erickson R, Scripture-Adams DD, Lesche R, Trumpp A, Zack JA, et al. Negative regulation of neural stem/progenitor cell proliferation by the Pten tumor suppressor gene in vivo. Science. 2001 Dec 7;294(5549):2186-9.

32. Groszer M, Erickson R, Scripture-Adams DD, Dougherty JD, Le Belle J, Zack $J A$, et al. PTEN negatively regulates neural stem cell self-renewal by modulating G0-G1 cell cycle entry. Proc Natl Acad Sci U S A. 2006 Jan 3; 103(1):111-6.

33. Otaegi G, Yusta-Boyo MJ, Vergaño-Vera E, Méndez-Gómez HR, Carrera AC, Abad $J$, et al. Modulation of the PI 3-kinase-Akt signalling pathway by IGF-I and PTEN regulates the differentiation of neural stem/precursor cells. J Cell Sci. 2006 Jul 1;119(Pt 13):2739-48.

34. Zheng $H$, Ying $H$, Yan $H$, Kimmelman AC, Hiller DJ, Chen A-J, et al. Pten and p53 converge on c-Myc to control differentiation, self-renewal, and transformation of normal and neoplastic stem cells in glioblastoma. Cold Spring Harb Symp Quant Biol. 2008;73:427-37.

35. Gregorian C, Nakashima J, Le Belle J, Ohab J, Kim R, Liu A, et al. Pten deletion in adult neural stem/progenitor cells enhances constitutive neurogenesis. J Neurosci Off J Soc Neurosci. 2009 Feb 11;29(6):1874-86.

36. Garcia-Junco-Clemente P, Golshani P. PTEN: a master regulator of neuronal structure, function, and plasticity. Commun Integr Biol. 2014 Jan 1;7(1): e28358
37. Veleva-Rotse BO, Barnes AP. Brain patterning perturbations following PTEN loss. Front Mol Neurosci. 2014;7:35.

38. Igarashi A, Itoh K, Yamada T, Adachi Y, Kato T, Murata D, et al. Nuclear PTEN deficiency causes microcephaly with decreased neuronal soma size and increased seizure susceptibility. J Biol Chem. 2018 15;293(24):9292-9300.

39. Tilot AK, Bebek G, Niazi F, Altemus J, Romigh T, Frazier TW, et al. Neural transcriptome of constitutional Pten dysfunction in mice and its relevance to human idiopathic autism spectrum disorder. Mol Psychiatry. 2016 Jan; 21(1):118-25.

\section{Publisher's Note}

Springer Nature remains neutral with regard to jurisdictional claims in published maps and institutional affiliations.
Ready to submit your research? Choose BMC and benefit from:

- fast, convenient online submission

- thorough peer review by experienced researchers in your field

- rapid publication on acceptance

- support for research data, including large and complex data types

- gold Open Access which fosters wider collaboration and increased citations

- maximum visibility for your research: over $100 \mathrm{M}$ website views per year

At BMC, research is always in progress.

Learn more biomedcentral.com/submissions 